\title{
WHO EXPERT COMMITTEE ON MALARIA
}

Twentieth Report

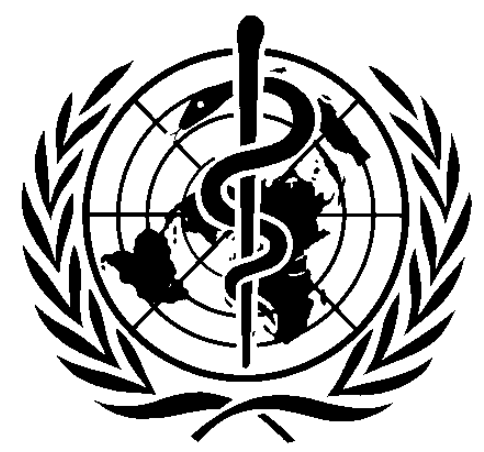

World Health Organization 
The World Health Organization was established in 1948 as a specialized agency of the United Nations serving as the directing and coordinating authority for international health matters and public health. One of WHO's constitutional functions is to provide objective and reliable information and advice in the field of human health, a responsibility that it fulfils in part through its extensive programme of publications.

The Organization seeks through its publications to support national health strategies and address the most pressing public health concerns of populations around the world. To respond to the needs of Member States at all levels of development, WHO publishes practical manuals, handbooks and training material for specific categories of health workers; internationally applicable guidelines and standards; reviews and analyses of health policies, programmes and research; and state-ofthe-art consensus reports that offer technical advice and recommendations for decision-makers. These books are closely tied to the Organization's priority activities, encompassing disease prevention and control, the development of equitable health systems based on primary health care, and health promotion for individuals and communities. Progress towards better health for all also demands the global dissemination and exchange of information that draws on the knowledge and experience of all WHO's Member countries and the collaboration of world leaders in public health and the biomedical sciences.

To ensure the widest possible availability of authoritative information and guidance on health matters, WHO secures the broad international distribution of its publications and encourages their translation and adaptation. By helping to promote and protect health and prevent and control disease throughout the world, WHO's books contribute to achieving the Organization's principal objective — the attainment by all people of the highest possible level of health.

The WHO Technical Report Series makes available the findings of various international groups of experts that provide WHO with the latest scientific and technical advice on a broad range of medical and public health subjects. Members of such expert groups serve without remuneration in their personal capacities rather than as representatives of governments or other bodies; their views do not necessarily reflect the decisions or the stated policy of WHO. An annual subscription to this series, comprising about 10 such reports, costs Sw. fr. 132.- (Sw. fr. 92.40 in developing countries). 
This report contains the collective views of an international group of experts and does not necessarily represent the decisions or the stated policy of the World Health Organization

\section{WHO EXPERT COMMITTEE ON MALARIA}

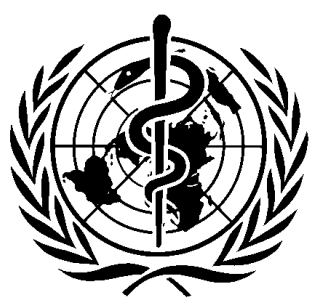


WHO Library Cataloguing-in-Publication Data

WHO Expert Committee on Malaria (1998 : Geneva, Switzerland)

WHO Expert Committee on Malaria : twentieth report.

(WHO technical report series ; 892)

1.Malaria-prevention and control 2.Malaria-drug therapy 3.Health care reform 4.Drug resistance 5.DDT-pharmacology I.Title II.Series

ISBN 9241208929 (NLM Classification: WC 765)

ISSN 0512-3054

The World Health Organization welcomes requests for permission to reproduce or translate its publications, in part or in full. Applications and enquiries should be addressed to the Office of Publications, World Health Organization, Geneva, Switzerland, which will be glad to provide the latest information on any changes made to the text, plans for new editions, and reprints and translations already available.

\section{(c) World Health Organization 2000}

Publications of the World Health Organization enjoy copyright protection in accordance with the provisions of Protocol 2 of the Universal Copyright Convention. All rights reserved.

The designations employed and the presentation of the material in this publication do not imply the expression of any opinion whatsoever on the part of the Secretariat of the World Health Organization concerning the legal status of any country, territory, city or area or of its authorities, or concerning the delimitation of its frontiers or boundaries.

The mention of specific companies or of certain manufacturers' products does not imply that they are endorsed or recommended by the World Health Organization in preference to others of a similar nature that are not mentioned. Errors and omissions excepted, the names of proprietary products are distinguished by initial capital letters.

Typeset in Hong Kong

Printed in Singapore

99/12601 - Best-set/SNP — 8000 


\section{Contents}

1. Introduction 1

2. Current global malaria situation 3

3. Implementation status of the Global Malaria Control Strategy 7

3.1 Provision of early diagnosis and prompt treatment 7

3.2 Implementation of selective and sustainable preventive measures 9

3.2.1 Vector control 9

3.2.2 Environmental management 10

3.2.3 Biological control: use of larvivorous fish 10

3.2.4 Chemoprophylaxis 10

3.3 Early detection, containment or prevention of malaria epidemics 11

3.4 Capacity building 11

3.5 Prevention of the re-emergence of malaria 12

4. Relationship of malaria control programmes to health sector reforms 12

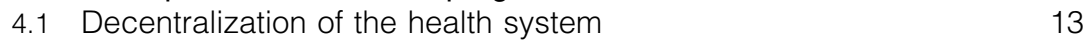

4.2 Health-care financing reforms 14

4.3 Increased partnership with communities and the private sector 16

5. Disease management 16

$\begin{array}{ll}\text { 5.1 Management of uncomplicated malaria } & 17\end{array}$

$\begin{array}{ll}\text { 5.1.1 Diagnosis } & 18\end{array}$

5.1.2 Availability and quality of treatment 24

5.2 Management of severe malaria at the periphery 25

6. Drug resistance of malaria parasites $\quad 27$

$\begin{array}{ll}6.1 \text { Monitoring of drug resistance } & 27\end{array}$

$\begin{array}{ll}\text { 6.1.1 Monitoring of therapeutic efficacy } & 27\end{array}$

$\begin{array}{ll}6.1 .2 \text { In vitro susceptibility testing } & 29\end{array}$

6.1.3 Surveillance-based molecular markers of drug resistance $\quad 29$

6.2 International monitoring of the drug response of $P$. falciparum 30

$\begin{array}{ll}\text { 6.3 Antimalarial treatment policy } & 30\end{array}$

$\begin{array}{ll}\text { 6.4 Containment of parasite drug resistance } & 31\end{array}$

7. Malaria epidemics: prediction, preparedness and control 32

7.1 Epidemic risk and epidemic-prone areas 32

7.2 Epidemic preparedness, prediction and prevention of epidemics 33

$\begin{array}{lll}7.3 & \text { Epidemiological information systems } & 34\end{array}$

$\begin{array}{ll}7.4 & \text { Epidemic response } \\ 7.5 & 35\end{array}$

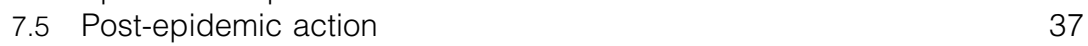

8. Prevention of malaria $\quad 37$

$\begin{array}{lll}8.1 & \text { Selective vector control } & 37\end{array}$

8.1.1 Indoor residual spraying 38

8.1.2 Use of DDT in malaria control 38

8.1.3 Use of insecticide-treated materials 39

8.1.4 Management of malaria in development projects 40 
8.1.5 New developments in Africa $\quad 41$

8.1.6 Use of gametocytocidal drugs $\quad 42$

8.2 Chemoprophylaxis 42

8.2.1 Chemoprophylaxis among non-immune people visiting
endemic areas

8.2.2 Prevention of malaria in pregnancy $\quad 42$

8.3 Malaria vaccines and basic research 44

8.4 Trends in insecticide resistance 44

8.5 Cost-effectiveness of preventive measures 46

9. Information systems and operational research 46

9.1 Epidemiological indicators 46

9.1.1 Standardized case definitions $\quad 46$

9.1 .2 Indicators $\quad 48$

9.2 Operational research $\quad 50$

9.2.1 National programme capabilities for operational research 51

9.2.2 Constraints on operational research 51

9.2.3 Addressing the constraints 52

9.2.4 Priority areas for operational research related to
programme objectives and policy

10. Award of the Darling Medal and Prize 55

11. Roll Back Malaria 56

12. Conclusions and recommendations $\quad 57$

$\begin{array}{ll}12.1 \text { Conclusions } & 57\end{array}$

$\begin{array}{ll}12.2 \text { Recommendations } & 59\end{array}$

12.2.1 General recommendation $\quad 59$

12.2.2 Relationship of malaria control programmes with
changing health sector reforms

12.2.3 Disease management 60

12.2.4 Drug resistance 60

12.2.5 Operational systems and operational research 61

12.2.6 Malaria in pregnancy 61

12.2.7 Vector control $\quad 62$

12.2.8 DDT 63

12.2.9 Epidemics 63

$\begin{array}{ll}\text { Acknowledgements } & 64\end{array}$

$\begin{array}{ll}\text { References } & 65\end{array}$

Annex 1

$\begin{array}{ll}\text { Use of DDT in vector control } & 69\end{array}$

Annex 2

$\begin{array}{ll}\text { Improving information exchange } & 71\end{array}$ 


\section{WHO Expert Committee on Malaria}

Geneva, 19-27 October 1998

\section{Members}

Dr R.B. Biritwum, Professor, Department of Community Health, Ghana Medical School, Accra, Ghana

Dr B.M. Greenwood, Professor, Department of Medical Parasitology, London School of Hygiene and Tropical Medicine, London, England

Dr P.F. Guillet, Head, Laboratory of Insect Pests, Institute for Research and Development, Montpellier, France

Dr Krongthong Thimasarn, Director, Malaria Division, Department of Communicable Disease Control, Ministry of Public Health, Nonthaburi, Thailand (ViceChairman)

Dr K. Marsh, Professor, Kenya Medical Research Institute (KEMRI) Centre for Geographic Medicine Research - Coast, Kilifi, Kenya (Rapporteur)

Dr K.M. Paluku, Director, Programme for Control of Communicable Diseases in Children, Kinshasa, Democratic Republic of the Congo

Dr T.K. Ruebush II, Chief, Malaria Epidemiology Section, Division of Parasitic Disease, Centers for Disease Control and Prevention, Atlanta, GA, USA

Dr W.H. Wernsdorfer, Professor, Institute of Specific Prophylaxis and Tropical Medicine, University of Vienna, Vienna, Austria (Chairman)

\section{Secretariat}

Mr Tarekegn Abose, Manager, Malaria and Other Vector-Borne Diseases Control, Ministry of Health, Addis Ababa, Ethiopia (Temporary Adviser)

Dr G. Barnish, Reader, Liverpool School of Tropical Medicine, Liverpool, England (Temporary Adviser)

Dr Le Dinh Cong, Director, Institute of Malariology, Parasitology and Entomology, Ministry of Health, Hanoi, Viet Nam (Temporary Adviser)

Dr A.L. El Idrissi, National Programme Manager, Department of Parasitic Diseases, Ministry of Health, Rabat, Morocco (Temporary Adviser)

Dr A.V. Kondrachine, Chief, Malaria Control, Division of Control of Tropical Diseases, WHO, Geneva, Switzerland (Secretary)

Dr Shiv Lal, Director, National Malaria Eradication Programme, Delhi, India (Temporary Adviser)

Dr J.A. Najera, Crans-près-Céligny, Vaud, Switzerland (Temporary Adviser) 


\section{Introduction}

The WHO Expert Committee on Malaria met in Geneva from 19 to 27 October 1998. The meeting was opened on behalf of the DirectorGeneral by Dr D.L. Heymann, Executive Director of the Communicable Diseases cluster. After describing the new organizational structure of WHO which was to be implemented in late 1998, he pointed out that during the last decade, considerable progress had been made in malaria control worldwide.

He noted that the previous meeting of the Expert Committee, held in 1989, was an important milestone in elaborating worldwide guidance for malaria control - at a time when the global malaria situation was seriously deteriorating. Most of Africa, south of the Sahara, continued to face an increasingly serious public health crisis as a result of the disease. Numerous civil wars and social unrest in various African countries, combined with fluctuating meteorological and ecological changes favourable to malaria transmission in areas that were previously malaria-free, also contributed to the problem. This was exacerbated by limited national financial resources, the absence of external support, inadequacies in technical guidance and insufficient experience in controlling malaria in highly endemic areas. The emergence and spread of chloroquine-resistant Plasmodium falciparum further aggravated the situation.

Although malaria occurred mainly in Africa, south of the Sahara, the spread of multidrug-resistant $P$. falciparum - which had developed in frontier areas in south-east Asia and South America during the 1980 s - threatened malaria control in other parts of the world.

In view of this serious situation, WHO's Executive Board in 1990 proposed that a Ministerial Conference on Malaria be held to encourage affected countries and the international community to intensify efforts to control the disease. After preparatory meetings involving representatives of donor agencies, research institutions, the United Nations and its specialized agencies, as well as managers, scientists and administrators, a consensus was reached on current standards for malaria control and a Global Malaria Control Strategy was formulated (1). The Global Strategy was presented to and endorsed by the Ministerial Conference on Malaria, which was held in Amsterdam, Netherlands, in October 1992.

In 1993, the Economic and Social Council of the United Nations initiated a review of the global malaria situation. This review was conducted in response to concerns expressed by members of the Council, especially those from countries in Africa where malaria was 
not receiving the urgent attention required from individual countries and the United Nations system. The Council's review led to the endorsement of the Global Strategy in the forty-ninth session of the United Nations General Assembly in December 1994. The General Assembly requested WHO to continue acting as the leading organization for malaria control, and to develop an action plan (for 1995 to 2000) in consultation with other United Nations agencies, other partners in malaria control and the affected countries. The Economic and Social Council of the United Nations endorsed the Action Plan in 1995 and called for increased resources for the prevention and control of malaria.

A further important development occurred in March 1996 when malaria was identified as a priority component of the United Nations system-wide Special Initiative on Africa. In June 1997, the Assembly of the Heads of State and Governments of the Organization of African Unity (OAU) adopted the Harare Declaration on Malaria Prevention and Control in the Context of African Economic Recovery and Development, pledging to consider malaria control as a priority. The Assembly reaffirmed its endorsement of the Global Malaria Control Strategy and the actions of WHO in its implementation, and called upon Member States to give full political support to malaria control, to develop and implement plans of action for malaria prevention and control, and to ensure well coordinated multisectoral action.

More recently, a Multilateral Initiative on Malaria in Africa (MIM) has been established, which is particularly concerned with strengthening research capability in Africa. This Initiative followed meetings in Senegal and the Netherlands in 1997 involving WHO, the World Bank, other United Nations agencies, the European Commission, the National Institutes of Health (the United States), the Wellcome Trust (the United Kingdom), and other donors as well as research workers from Africa and developed countries.

One of the most significant recent financial investments in malaria control, particularly in Africa, south of the Sahara, was the allocation of substantial funds by the Director-General of WHO to priority countries for 1997 and 1998. In 1998, the newly elected DirectorGeneral of WHO initiated the Roll Back Malaria project, thus establishing malaria as one of WHO's highest priorities.

The importance of malaria was also recognized by the members of the Group of Eight Summit held in 1998 in Birmingham, England, at which the British Government pledged $£ 60$ million towards the fight against malaria. A significant proportion of this money will be allocated to Roll Back Malaria. 


\section{Current global malaria situation}

At present, about 100 countries or territories in the world are considered malarious, almost half of which are in Africa, south of the Sahara (Fig. 1). Although this number is considerably less than it was in the mid-1950s (140 countries or territories), more than 2400 million of the world's population are still at risk.

The incidence of malaria worldwide is estimated to be $300-500$ million clinical cases each year, with about $90 \%$ of these occurring in Africa, south of the Sahara - mostly caused by P. falciparum. Malaria ${ }^{1}$ is thought to kill between 1.1 and 2.7 million people worldwide each year, of whom about 1 million are children under the age of 5 years in Africa, south of the Sahara. These childhood deaths, resulting mainly from cerebral malaria and anaemia, constitute nearly $25 \%$ of child mortality in Africa. Fatality rates of $10-30 \%$ have been reported among children referred to hospital with severe malaria, although these rates are even higher in rural and remote areas where patients have restricted access to adequate treatment. Deaths from malaria in countries outside Africa, south of the Sahara, occur principally in non-immune people who become infected with $P$. falciparum in areas where diagnosis and treatment are not available.

One of the greatest challenges facing malaria control worldwide is the spread and intensification of parasite resistance to antimalarial drugs. The limited number of such drugs has led to increasing difficulties in the development of antimalarial drug policies and adequate disease management.

Resistance of $P$. falciparum to chloroquine is now common in practically all malaria-endemic countries of Africa (Fig. 2), especially in east Africa, thus posing increasing problems for the provision of suitable treatment. As a consequence Malawi and Kenya, in 1993 and 1996 respectively, changed their recommendations for first-line treatment of uncomplicated malaria from chloroquine to sulfadoxine/pyrimethamine, and Botswana and South Africa revised their treatment guidelines in 1997. Resistance to sulfadoxine/pyrimethamine, the main alternative to chloroquine, is widespread in south-east Asia and South America. Recent reports from Kenya and the United Republic of Tanzania suggest that changes in parasite susceptibility to sulfadoxine/pyrimethamine have occurred and may presage clinical resistance. Mefloquine resistance is now common in the border areas of Thailand with Cambodia and Myanmar. Parasite sensitivity to

Either alone or in combination with other diseases. 


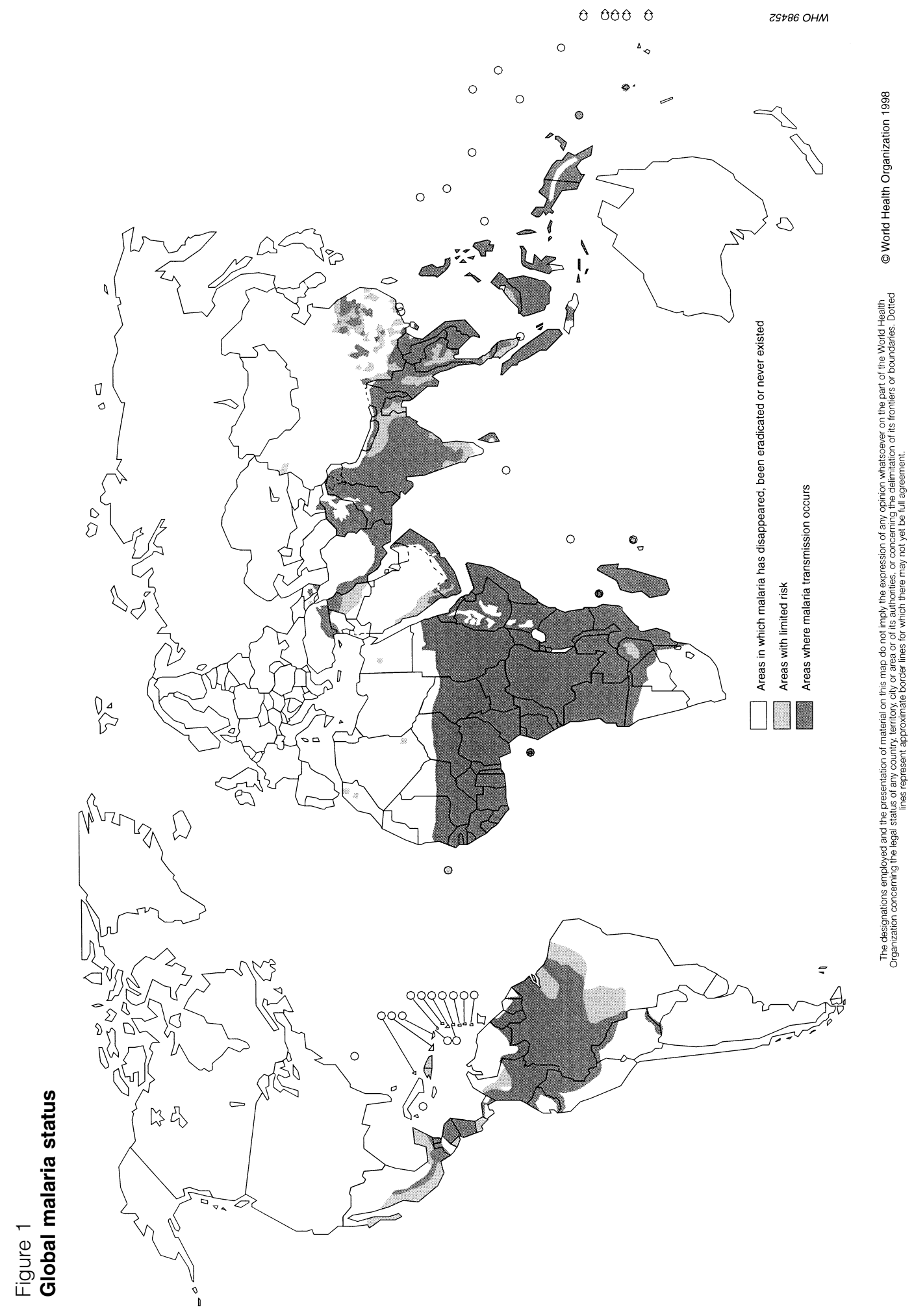




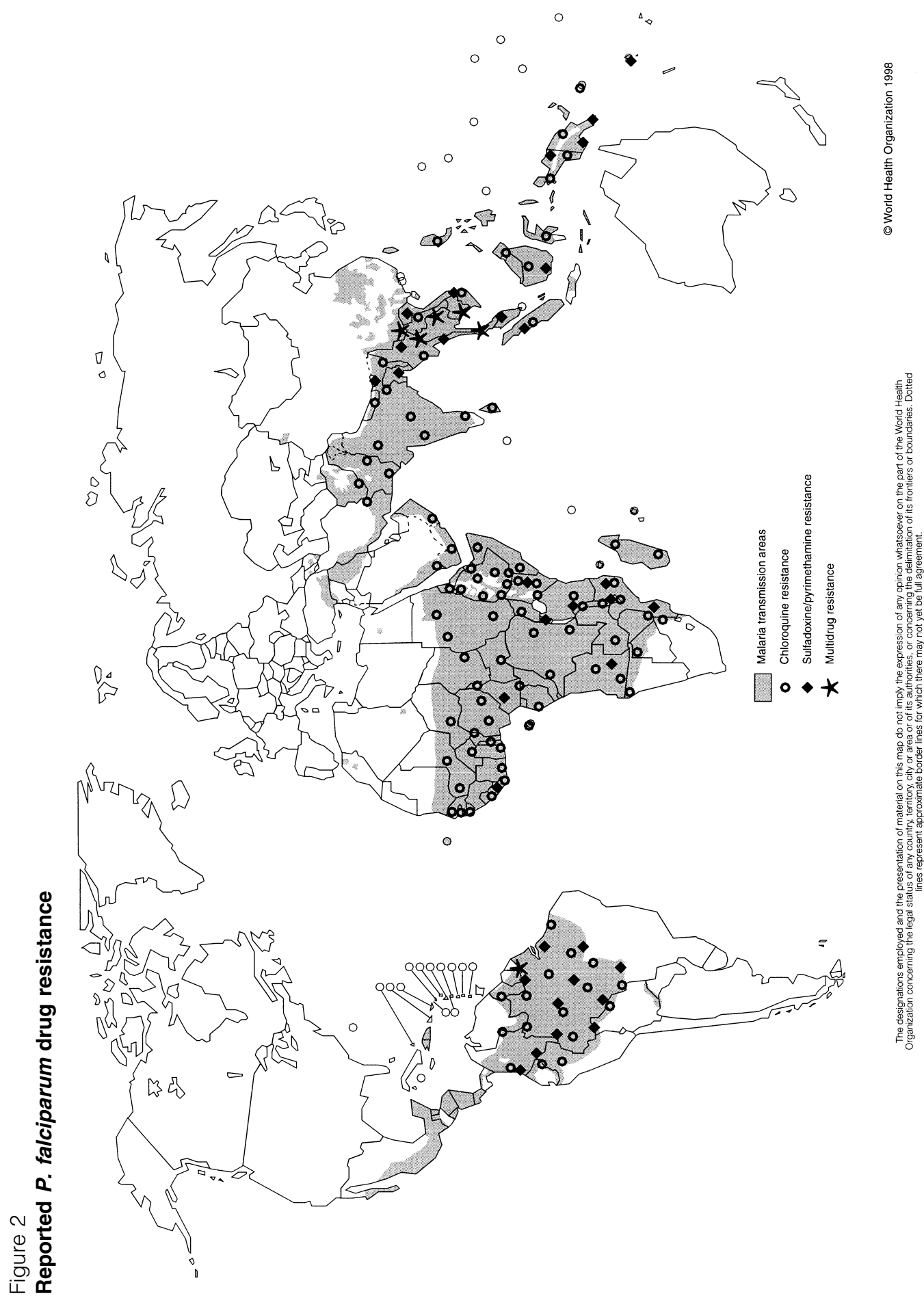


quinine is declining in several other countries of south-east Asia and in the Amazon region, where it has been used in combination with tetracycline for the treatment of uncomplicated malaria. Consequently, artemisinin and its derivatives are now increasingly being used as first-line treatment in some of these areas.

Resistance of $P$. vivax to chloroquine has now been reported from Indonesia (Irian Jaya), Myanmar, Papua New Guinea and Vanuatu. Cross-resistance to amodiaquine sometimes occurs.

An increasing number of malaria epidemics have been recently documented throughout the world, particularly in Africa. Areas become epidemic when conditions that normally limit transmission change radically as a result of abnormally heavy rains, long periods of increased humidity and temperature, or more permanent changes of microclimate due to the development of irrigation systems, agricultural projects or tree plantations.

Urban and periurban malaria are on the increase in south Asia and in many areas of Africa. Military conflicts and civil unrest, along with unfavourable ecological changes, have greatly contributed to malaria epidemics, as large numbers of unprotected, non-immune and physically weakened refugees move into malarious areas. Such population movements contribute to new malaria outbreaks and make epidemicprone situations more explosive.

The concentration of gold or gem mining and logging in the South American or south-east Asian forested regions, where the continuous flow of migrant workers are exposed to very high malaria transmission, also leads to epidemics. Moreover, these are the original foci of multidrug resistance outside Africa, south of the Sahara.

Another disquieting factor is the re-emergence of malaria in areas where it had been eradicated (e.g. Democratic People's Republic of Korea, Republic of Korea and Tadjikistan), or its increase in countries where it was nearly eradicated (e.g. Azerbaijan, northern Iraq and Turkey). The current malaria epidemics in the majority of these countries are the result of a rapid deterioration of malaria prevention and control operations, due to a variety of reasons.

The economic effects of malaria are especially noticeable in rural areas, where malaria frequently strikes at the time of year when there is greatest need for agricultural work. Furthermore, the disease is a common cause of school absenteeism, reaching as high as $28 \%$ in some places. The estimated annual direct and indirect cost of malaria in Africa alone is more than US\$2000 million. 


\section{Implementation status of the Global Malaria Control Strategy}

The Committee reviewed the progress made since 1992 in the implementation of WHO's Global Malaria Control Strategy, the four basic technical elements of which are:

- to provide early diagnosis and prompt treatment of malaria;

- to plan and implement selective and sustainable preventive measures, including vector control;

- to detect early, contain or prevent epidemics;

- to strengthen local capacities in basic and applied research to permit and promote the regular assessment of a country's malaria situation, in particular the ecological, social and economic determinants of the disease.

Additionally, an important consideration now is the prevention of the re-emergence of malaria in countries that had either succeeded in bringing down malaria incidence to a sporadic level, or had interrupted transmission altogether.

\subsection{Provision of early diagnosis and prompt treatment}

This element of the Global Strategy has been accepted and implemented by all malaria-endemic countries of the WHO regions as the cornerstone of their control efforts. In the WHO African Region, a significant development during the past five years has been the development of national plans of action, in line with the recommendations of the Global Strategy by the majority of countries in Africa, south of the Sahara. Retraining of more than 16000 health providers in the early diagnosis and appropriate treatment of malaria has been undertaken in many countries, laying the foundation for effective malaria control. In the WHO Western Pacific Region, there has been a large reduction in malaria mortality, especially in Viet Nam, due in part to the expansion of treatment facilities, and the use of artemisinin (distributed free of charge) and insecticide-impregnated bednets. In the WHO European Region, the Global Strategy has been implemented with the goal of providing radical cure. Despite this substantial progress, however, problems remain in each region.

In some countries of the WHO Eastern Mediterranean, South-East Asia and Western Pacific Regions, inadequate use of public sector facilities for the management of malaria is the rule because of the poor quality of these facilities. As a consequence, malaria is often managed in the private health-care sector where treatment guidelines are frequently not followed. In some countries, presumptive 
treatment of malaria persists and the continuing spread of parasite multidrug resistance remains a matter for concern. There have been many training programmes on clinical management of malaria but more are needed, particularly within the private sector.

In the WHO European Region, disruption of the traditional links between the newly independent states of the former Soviet Union has resulted in difficult economic conditions. The exodus of trained staff and a shortage of supplies have resulted in sudden and severe reductions in the quality of health care, including the diagnosis, management and prevention of malaria. In several western European countries, the fatality rate among imported falciparum malaria cases $(1.5-7.0 \%)$ is unacceptable and incompatible with the generally high standards of medical care in the region - suggesting that both diagnosis and management of the disease are inadequate.

In the WHO African Region, although coverage with treatment facilities has improved overall, problems remain in many countries, especially those affected by civil disturbances. The introduction of cost recovery for treatment in many countries has had variable consequences. In some areas, the introduction of costs has had an adverse effect on the management of malaria, especially when it has not been accompanied by any improvement in the quality of care provided. First- and second-line drugs of uncertain quality are in widespread use in both the public and private sectors, and few countries in the region have the resources to maintain surveillance of drug quality. The emergence of $P$. falciparum resistant to chloroquine and sulfadoxine/ pyrimethamine in eastern and western Africa is a matter of concern because of the much greater cost of alternative treatments.

In the WHO Region of the Americas, major advances have been made in integrating traditional malaria control programmes into the general health services. Although the efficiency of local health services in the detection of malaria cases is considerably higher than that of specialized services, it still requires strengthening in many countries. The epidemiological redefinition of malaria risk areas has resulted in the prioritization of areas and an improvement in the targeting of diagnostic and treatment facilities to those in greatest need.

The Committee gave particular attention to the review of three technical issues:

\section{- Management of severe malaria}

Although the management of severe malaria in health facilities has improved somewhat in many areas, problems remain, especially in 
Africa, south of the Sahara, where many patients with severe malaria live in areas where there is no transport system available to take them to hospital. In such circumstances, the provision of emergency pre-referral therapy, such as rectally administered artemisinins, may reduce mortality.

- Antigen detection tests in diagnosis

Rapid tests for the diagnosis of malaria based on "dipstick" technology (see section 5.1.1) are being used frequently in western Europe for visitors returning from malaria-endemic countries. In India, they are being used selectively in both the public and private sectors. Such tests are likely to prove of particular value in the early detection of epidemics and of malaria in other emergency situations.

\section{- Drug resistance}

The expanding problem of drug resistance continues to challenge malaria control efforts based on early diagnosis and treatment. New antimalarial drugs and approaches to overcome parasite resistance are needed to deal successfully with this challenge.

\subsection{Implementation of selective and sustainable preventive measures}

\subsubsection{Vector control}

The extent of implementation of vector control measures varies widely among the WHO regions.

Use of insecticide-treated materials

Insecticide-treated materials have been successfully and safely used to control malaria morbidity and mortality in a range of environments throughout the African and Western Pacific Regions. In Africa, south of the Sahara, use of insecticide-treated mosquito nets and other materials is gradually increasing, with a shift from project-based to operational implementation. Programmes based on the use of insecticidetreated materials have been successfully implemented in many areas of the WHO Western Pacific Region. In Viet Nam, bednets are widely and successfully used as a component of the national malaria control strategy, including in epidemic-prone areas; in China, millions of such nets have been used routinely for many years; in the Solomon Islands, the use of bednets has sharply increased over the past few years, resulting in a dramatic reduction in malaria. The general experience is that this intervention, along with appropriate education, is highly acceptable, even in populations that do not traditionally use bednets.

The Committee recognized, however, that many problems remain before the full potential of this control tool can be realized. These 
include the difficulty of ensuring that nets are available to all who need them, in particular the underprivileged. The fact that only one class of insecticide (pyrethroids) can currently be used to treat nets and that resistance to pyrethroids has already been recorded among anopheline mosquitos is disturbing. In addition, the long-term efficacy of this control measure has not yet been determined.

\section{Indoor residual spraying with insecticides}

In many programmes, mainly in the Americas and some countries in Asia, indoor residual spraying continues to be the main vector control measure implemented. There is, however, a tendency to reduce reliance on spraying, and there is also a marked decrease in the use of conventional residual insecticides, such as DDT, which have been replaced by new-generation insecticides, such as the pyrethroids, at considerable financial cost to the programmes. Improvement in local knowledge of malaria epidemiology (especially disease transmission), with refinement of surveillance mechanisms and proper use of collected data, could lead to the implementation of more selective vector control and further reduce reliance on indoor residual spraying.

\subsubsection{Environmental management}

Man-made malaria, which has been called the "curse of the tropics", continues to be a very frequent sequel to economic development projects in malarious areas throughout the world. The disease arises not only as a result of the ecological disturbance that these projects produce, particularly those dealing with hydrological resources and agriculture, but also from the resulting mix of populations, whose members differ in their malaria immunity status.

\subsubsection{Biological control: use of larvivorous fish}

Larvivorous fish have been demonstrated to have potential in certain situations (e.g. in epidemic-prone areas of Karnataka State in India) but not yet on an operational scale.

\subsubsection{Chemoprophylaxis}

In pregnancy

Few women resident in malaria-endemic areas currently receive malaria chemoprophylaxis. In some countries, national guidelines recommend the provision of chemoprophylaxis during pregnancy but little effort is made to implement this measure. In other countries, chemoprophylaxis with chloroquine is available, but is only weakly effective because of lack of patient compliance and $P$. falciparum 
drug resistance. There are at present no fully effective and feasible approaches to prevent malaria in non-immune pregnant women in endemic or epidemic-prone areas. It has been shown that infection with $P$. vivax also adversely affects the outcome of pregnancy, and the role of chemoprophylaxis and/or intermittent treatment in the management of this infection also needs to be examined.

\section{In children}

Chemoprophylaxis is generally not recommended for children who are likely to have lifelong exposure to malaria.

\subsection{Early detection, containment or prevention of malaria epidemics}

There is a growing realization of the need to implement programmes to predict and prevent malaria epidemics, and a few countries have already started to develop epidemic risk monitoring. Nevertheless, in the majority of countries, the delimitation of epidemic-prone areas and situations has not yet been fully undertaken, resulting in epidemic detection usually being carried out with unacceptable delays. Experience has shown that available and simple indicators (e.g. meteorological data and population movements) are not yet routinely used for predicting and preventing epidemics. In most countries, there is insufficient collaboration with other sectors, particularly the meteorological and agriculture services. This lack of intersectoral collaboration is delaying the establishment of an efficient prediction system.

\subsection{Capacity building}

Capacity building for basic and applied research and for evaluation of malaria control programmes has been considered an essential part of the Global Strategy for Malaria Control. Some progress has been made since the previous meeting of the Expert Committee, but more needs to be made. Serious shortages of skilled personnel, especially at senior level, exist in some regions, especially the African Region. As the Global Strategy has moved more towards selective malaria control based on sound local knowledge of the epidemiology of the disease, so the need for malaria expertise is becoming more pressing. Although this shortage of trained personnel is most severe in malariaendemic countries, it is also seen outside endemic areas, in countries whose nationals have, in the past, played an important part in malaria control and whose specialists are still asked for advice. In some areas there is no overall shortage of trained staff, but they cannot fulfil their potential because of an inappropriate career structure, inadequate salary or a lack of logistic resources. In some cases, trained personnel 
have been forced to take up appointments in areas that do not use their expertise. It might be possible to recruit some of these scientists back into malaria control, perhaps after some retraining, if their conditions of service were improved.

\subsection{Prevention of the re-emergence of malaria}

The Committee noted that most of the countries that had achieved malaria eradication were able to maintain this status. Additionally, a number of countries have succeeded in eliminating $P$. falciparum, while transmission of $P$. vivax still continues (e.g. Iraq, Morocco and Syrian Arab Republic). In these countries, appropriate measures should be planned to monitor the reintroduction of malaria and to recognize episodes of renewed transmission, and to implement appropriate control measures.

In a number of countries, the incidence of malaria has been brought down to such low levels that total interruption of transmission may be a feasible objective (e.g. Algeria, Egypt, Morocco, Oman and United Arab Emirates). For such countries, interventions could be planned to achieve complete interruption of transmission based on the principles of eradication.

\section{Relationship of malaria control programmes to health sector reforms}

In response to political and economic demands, health sector reforms are underway in many countries, with the overall aim of improving efficiency in the management and use of health resources. Such reforms are not expected to impair the quality of service delivery and coverage, but instead, by engaging in partnerships with other sectors (e.g. government education and environment departments, food and drug regulatory boards, and the agricultural sector), private healthcare providers and communities, they should finally lead to important improvements in the quality of care.

In most countries, health sector reforms have included:

- organizational reforms - restructuring of the ministry of health and decentralization of planning, budgeting authority, control of financial resources and responsibility for implementation of programme activities;

- health financing reforms - cost sharing, user fees, and public and private health insurance mechanisms;

- increased partnerships with communities and private health-care providers. 


\subsection{Decentralization of the health system}

Decentralization, an essential strategy in the implementation of health sector reform, involves the transfer of resources and authority to district and sub-district levels. It should also empower local authorities and communities to identify their priorities and needs. Health issues should be addressed directly through enhanced community awareness and knowledge about disease prevention, diagnosis and treatment, as well as through local operational research activities.

The poorly managed decentralization of malaria control programmes has undermined their effectiveness and hampered their ability to fulfil their responsibilities. As a result of this decentralization, which often occurs without any input from malaria control programme staff, programmes that were previously highly effective have lost direction and focus. In addition, experienced and dedicated personnel with specific technical expertise have retired or moved to other positions. Thus, programmes have lost the capability to respond rapidly and effectively to changing conditions (e.g. epidemics), and their commitment and ability to guide operations at the community level.

These losses have generally not been counterbalanced by the creation and development of technical competence and resources at the district and sub-district level, thus creating a vacuum in malaria control expertise and implementation. As a result, many of the potentially positive outcomes of decentralization, such as local ownership and responsibility for the programme, more active community involvement, increased ability to tailor control measures to local epidemiological situations, and greater intersectoral collaboration, are not being seen. Similar problems have also been observed in countries which lacked an effective national control programme when the decentralization process began: such countries are left disorientated and unable to deal effectively with the challenge of building up human resources and technical competence at the central and local levels.

Effective management of malaria control activities requires that certain national-level competence and coordinating functions be retained or developed at the central level of the programme (not necessarily the nation's capital) during the process of decentralization. These functions include providing strategic direction to the programme, developing malaria policy at the national level, and setting standards, norms and indicators for monitoring the progress of operational activities. In addition, they include mobilizing and coordinating external funding, epidemiological analysis, quality assurance, providing technical training and support for malaria team members at the local level, coordinating the response to epidemics, and evaluating and validating 
programme activities, including operational research undertaken at the district level. Thus, effective management of control programmes will require not only expertise in disease management, epidemiology, surveillance and vector control, but also social, economic and behavioural studies and adequate administrative, statistical and logistic support. In countries where malaria is a high priority within the ministry of health, part-time staff who do not work exclusively on control programmes cannot normally provide this level of competence.

Important benefits of a decentralization policy to malaria control are that decision-making and planning capacity would be based at the level where the problems occur. At the district and sub-district levels, where responsibility for the successful execution of control activities is centred, functions required would include local-level planning, resource allocation, disease surveillance, monitoring of programme activities, health education, training and vector control. District staff must serve as an effective liaison between field staff and national-level staff and provide continuous operational guidance to the peripheral level. Epidemiological information collected at the periphery should be initially analysed at this level, with feedback and technical guidance provided to field staff for rapid action, while the central team is provided with a summary of the data on a regular basis for more detailed analysis. Local capacity for surveillance and rapid response needs to be strengthened, especially in high-risk and epidemic-prone areas.

It is crucial that responsibility for the implementation of malaria control activities at the district and sub-district levels be accompanied by adequate funding. Furthermore, sufficient logistic support must be provided to enable local authorities and staff to carry out their responsibilities and respond rapidly and effectively to changes in the epidemiological situation.

\subsection{Health-care financing reforms}

The escalating costs of health care and the inability of governments to keep pace with them have been the driving force behind efforts at cost recovery through cost sharing, user fees and health insurance. While these reforms have contributed to more efficient use of resources, improved availability of drugs at peripheral level and the generation of profits which can be invested in other local priority projects, they have also resulted in an increasing proportion of health-care costs being borne by the poor.

Since guaranteed access to early diagnosis and appropriate treatment of febrile illnesses is the key to reducing malaria morbidity and mor- 
tality, any change which might influence the provision of prompt and effective treatment is of critical importance to malaria control efforts. Experience has shown that if the cost of treatment increases through some form of user fees without any change in the quality of health care, attendance at health facilities will fall and this will affect the poor disproportionately. If, however, increased costs lead to improvement in the quality of care, use of the facilities does not necessarily decrease. An analysis of the impact of increased treatment costs on the provision and quality of care in the "Bamako Initiative", a largescale primary health-care project in Africa, south of the Sahara, involving community health financing through a revolving fund for drugs, showed mixed results. In some cases, the availability and demand for drugs increased, while in others, it was possibly a disadvantage for the poor.

Thus at present, there is no evidence that cost recovery per se has resulted in improved quality of care. In fact, as costs are raised, greater proportions of patients tend to seek antimalarial treatment from private or informal sources, where they are typically treated with inappropriate drugs or incorrect dosages. This may result in increased morbidity and mortality. There is evidence from some countries that user fees have also promoted irrational prescribing practices in the treatment of malaria, such as subcurative treatments, polypharmacy (i.e. prescribing multiple drugs), concomitant administration of antibiotics and/or steroids, and the use of injections when they are not indicated.

In spite of efforts to introduce health finance reforms for curative services, most people would agree that preventive services should be managed differently. Activities such as the Expanded Programme of Immunization and provision of antenatal and postnatal preventive services have been viewed as priorities for public financing. It has always been accepted that indoor residual spraying should be publicly financed, and it has also been suggested that chemoprophylaxis or intermittent treatment for malaria in pregnancy should be provided free of charge. The situation is, however, less clear with regard to bednets and other insecticide-treated materials. It has been argued that, given the disproportionate impact of falciparum malaria on pregnant women and young children (under 5 years) in Africa, south of the Sahara, these groups should be provided with free insecticidetreated bednets as part of an antenatal or postnatal care package. While this may seem attractive, experience has shown that this may not be the best way to target these populations, as bednets often end up being used by family members other than those for whom they were intended, or sold to others. Therefore, it may be more effective 
to provide insecticide-treated materials to everyone at a subsidized price.

There is a need for national malaria control programmes to become more proactive in defining their objectives for curative and preventive care as health-care financing reforms take place. This will ensure that the population groups most affected by malaria are not placed at a disadvantage because they have to assume a disproportionately large share of the cost of providing good-quality health services. Guaranteed access to early diagnosis and treatment of febrile illnesses is an essential component of malaria control efforts. This could be accomplished if control programmes move towards the goal of providing free diagnostic and treatment services to all.

\subsection{Increased partnership with communities and the private sector}

The costs of extending public health services to the community level have accelerated the process of involvement by communities and the private sector as partners in malaria control. This can be expected to be a slow but continuous process. So far, information is becoming available, largely from Africa, south of the Sahara, on the cost-effectiveness of three types of interventions undertaken at the community level: the provision of widely available curative treatment, usually by village volunteers; chemoprophylaxis for pregnant women; and the use of insecticide-treated materials. Information is needed from different epidemiological settings on the simultaneous use of such interventions.

While community-based interventions offer hope for the future, it is difficult at present to draw conclusions about the types of interventions that could successfully be delivered using community involvement. In spite of evidence that early treatment of malaria in children lowers case-fatality rates, it has been difficult to design communitybased distribution mechanisms that demonstrate an impact on mortality. While it is clear that insecticide-treated materials for all and chemoprophylaxis for pregnant women would save lives and are costeffective in research settings, it is not yet known whether they can be delivered at low cost in a practical programme setting. Simultaneous use of two or more of these interventions, which may be possible as community involvement increases, offers promise of a synergistic effect on severe morbidity and mortality.

\section{Disease management}

Disease management remains a fundamental and indispensable element of malaria control. Its aims are to: 
- avoid the progression of mild malarial disease to severe or complicated disease;

- prevent death or sequelae from severe and complicated malaria;

- limit the duration of disease;

- prevent transmission of malaria in certain situations, especially where transmission is low;

- minimize the risk of selection and spread of drug-resistant parasites.

\subsection{Management of uncomplicated malaria}

The management of uncomplicated malaria involves patients, carers, health-care staff, drug vendors, including pharmacists and drug suppliers. Ideally, the management of this disease includes:

- recognition of signs and symptoms which could be caused by malaria;

- seeking care;

- diagnosis of malarial disease and/or other febrile conditions;

- referral to a higher level of care, if necessary;

- prescription of correct treatment;

- education of the patient or carer on:

- how to take or administer the drugs

- the expected result of treatment

- when to return to the health facility

- danger signs

- side-effects

- prevention of malaria;

- dispensing or selling the correct drugs of assured quality, with the first dose always being taken under supervision;

- patient compliance with prescription instructions;

- follow-up to check whether the expected therapeutic effect has been achieved.

It is the responsibility of malaria control programmes and essential drug programmes within the general health services to ensure that the best possible quality of disease management is available to patients. The crucial concern is the timeliness of correct treatment with the correct drug. As mild falciparum malaria can progress to severe disease in a few hours, especially in children, effective treatment for the disease must be available close to the home and must be complete.

The elements of disease management are discussed more fully elsewhere (2-4). Because of recent technical advances and ongoing health sector reforms, the issues of greatest interest to malaria control are currently diagnosis, choice of treatment and availability of drugs. 


\subsubsection{Diagnosis}

The diagnosis of malaria can be based on clinical criteria and/or techniques for parasite detection.

\section{Clinical diagnosis}

As most cases of malaria are still treated on the basis of clinical diagnosis, it is inevitable that many patients without the disease receive antimalarial drugs. This causes unwarranted costs for patients and/or health services, risks of side-effects, and if the drugs are longacting, this may lead to the selection of resistant parasite strains. Conversely, lack of sensitivity of clinical criteria may cause some patients suffering from malaria not to be treated, thereby risking progression to severe disease and death.

While it is important to establish clinical diagnostic criteria which minimize the number of patients who receive the wrong treatment, improving diagnostic sensitivity has high priority - given the high case-fatality rate of untreated falciparum malaria. A full assessment of the patient is always needed, since simply equating fever with malaria may lead to other curable conditions being overlooked.

Unfortunately, the signs and symptoms of malaria, such as fever, chills, headache and anorexia, are generally nonspecific. Recent studies have identified some signs and symptoms which, especially in combination, have diagnostic value in specific epidemiological and operational situations (5-7). However, it is not possible to apply any one set of clinical criteria to the diagnosis of all types of malaria in all patient populations. Experience has shown that clinical diagnostic criteria must vary from area to area according to the intensity of transmission, the prevalent species of malaria parasite, the incidence of other causes of fever (e.g. dengue), the qualifications of health-care providers, and health-service infrastructure.

In general, in settings where the risk of malaria is low (i.e. in areas of low endemicity or, where malaria is highly seasonal, during the lowtransmission season), clinical diagnosis of uncomplicated malaria is based on the degree of exposure to malaria, and a history of fever ${ }^{1}$ with no features of other severe diseases. The absence of an alternative explanation for the fever has been recommended but, according to most studies, this will exclude only a small proportion of patients with fever from unnecessary treatment (8). The Integrated Management of Childhood Illness materials (2) provides clinical criteria,

\footnotetext{
In this context, "history of fever" means the presence of fever and/or a history of fever within the past 4 days.
} 
based on data from Africa, for treatment of children in low-risk settings. These are fever, without a runny nose or measles, and no other apparent cause of the fever. However, there is a need for more local clinical epidemiological research on specific criteria for the diagnosis of falciparum malaria in both children and adults, and for vivax malaria.

In settings where the risk of malaria is high (i.e. in areas where malaria transmission is stable or during the high-transmission season of seasonal malaria), the accepted criteria for the treatment of malarial disease in young children (generally below 5 years, but this depends on the level of endemicity) and pregnant women are a history of fever or the presence of clinically detectable anaemia, for which pallor of the palms seems to be the most reliable sign in young children (9). In older children, adult males and non-pregnant women, the sole criterion is a history of fever. The incidence of malarial disease in these age groups is generally low, and therefore the absence of an alternative explanation for the fever may be useful, especially in drawing attention to other conditions that may need to be treated.

In malaria epidemics, all patients with a history of fever should normally be treated for the disease.

\section{Diagnosis based on parasite detection ${ }^{1}$}

Microscopical or other parasite detection tests are an important supplement to clinical observation. However, in areas with high and stable transmission, asymptomatic parasite carriers may be common. If microscopy is available, the diagnosis of uncomplicated malaria is normally based on a history of fever and a positive blood slide. Malarial disease may exist despite a negative microscopy result, but unless the patient has already started treatment and there is a high clinical suspicion of the disease, treatment for uncomplicated malaria should not be given, as long as blood slides are negative.

Given the low specificity of all clinical case definitions, there is a compelling need to make parasite detection more widely available. Basic microscopy has many advantages: it has low direct costs, it is sensitive, and it can be used to differentiate between species and determine parasite density levels; it can also be used to diagnose many other conditions. However, experience in malaria-endemic areas has shown that, for a variety of operational reasons, it can be difficult to maintain good microscopy at the periphery of the health services where most patients are treated. In some settings where the numbers

\footnotetext{
Parasite detection is used here as a common term for microscopical examination of blood for Plasmodium species and immunological techniques for detection of plasmodial antigens in blood.
} 
of patients are very high, it may be warranted to restrict microscopical analysis to severely affected patients and suspected treatment failures.

The detection of malaria parasites using the quantified buffy coat (QBC) technique is easy to learn, has high sensitivity and specificity and is quicker to perform than standard microscopy. However, this technique requires specialized equipment and consumables, making it prohibitively expensive. It is therefore unlikely to be used by health services in the majority of endemic countries.

Among the available antigen-based tests ("dipsticks"), those based on P. falciparum histidine-rich protein II (HRPII) detection have been most extensively investigated under field conditions. Such tests have good sensitivity for asexual $P$. falciparum, with a detection limit of 60 asexual parasites per microlitre (10), although expert microscopy can detect as few as 4 asexual parasites per microlitre. However, the quality of microscopy is sometimes so poor that microscopy cannot be used as a basis for diagnosis. In such circumstances, antigen detection tests are often at least as sensitive and specific as routine microscopy at the periphery of the health services.

The acceptance of antigen test results as a basis for decisions on whether to give treatment depends on the parasite density threshold for disease in the patient population. One problem with HRPII-based tests is the persistence after effective treatment of HRPII antigenaemia (11), possibly making these tests less suitable for identifying treatment failures. Newer tests based on the detection of parasitespecific lactate dehydrogenase have the advantage that they can detect other species than $P$. falciparum, and they are not persistently positive after effective treatment (12). Although they have not yet been as extensively field-tested as the HRPII-based tests, they are commercially available.

Dipstick tests are already in use in certain countries (e.g. Brazil, Cambodia and India). These tests can be learnt within a few days and are easy for clinical and paraclinical staff to use in peripheral health facilities. The main obstacle to their widespread use in health care in malaria-endemic areas is their cost, which is still more than US\$ 1 per test. As the price of first-line treatment goes up, and - it is hoped the price of dipstick tests goes down, it is likely that these tests will become widely available as an effective and appropriate tool for improved targeting of antimalarial drugs to the patients who need them.

Research on operational issues associated with antigen detection tests and microscopy, including cost-effectiveness and willingness to pay, is of great importance in areas where there is need for improvement of the current practice of clinical diagnosis. 


\section{Treatment of uncomplicated malaria}

The principles of treatment of malaria are well described $(4,13,14)$. In many parts of the world, the selection of first-line treatment for uncomplicated malaria is extremely difficult because of increasing drug resistance of $P$. falciparum and, more recently in some areas, of $P$. vivax. The factors to be taken into account (15) are:

- drug effectiveness (which is determined mainly by efficacy (see below), patient compliance and drug quality);

- drug side-effects, tolerance, costs, affordability, availability and acceptability to the consumer and provider;

- characteristics of the health services.

In the decision-making process, it is essential to define efficacy by clarifying the objective of treatment. There are basically two situations in which the objectives will differ:

- Where malaria transmission is intense, most patients are semiimmune and asymptomatic infections are common. The objective of treatment is to obtain a clinical cure (i.e. clearance of signs and symptoms, but not necessarily of parasites). Antimalarial treatment must be schizonticidal but not necessarily gametocytocidal.

- Where transmission is unstable, there is no significant immunity and asymptomatic infections are rare. The objective of treatment is to obtain a radical cure, since clinical cure can rarely be achieved without parasite clearance. In certain circumstances, it may be necessary to reduce the reservoir of infection by supplementing schizonticidal treatment of falciparum malaria with a gametocytocidal drug, usually primaquine in a single dose (4). The public health benefits must be weighed against the small risk of side-effects and the cost-effectiveness of such a measure.

At present, chloroquine is still the preferred first-line treatment for malaria caused by any Plasmodium species sensitive to the drug. When a patient population with falciparum malaria does not respond satisfactorily to chloroquine, the treatment of choice is sulfadoxine/ pyrimethamine or amodiaquine, although it must be remembered that cross-resistance between chloroquine and amodiaquine has been reported (16). (Criteria for a satisfactory response are described in section 6.)

Ideally, the first dose of the drug should be given under observation. In the case of a single-dose treatment, such as sulfadoxine/ pyrimethamine, this will ensure adequate treatment. With a multipledose regimen, it will provide an opportunity to educate the carer and patient about correct treatment. 
When the response to sulfadoxine/pyrimethamine and amodiaquine is no longer satisfactory, the choice of treatment becomes difficult. Despite its price and side-effects, mefloquine would be the most appropriate choice because of the simplicity of administration in one or two doses. However, use of mefloquine as first-line treatment in areas with intense transmission (e.g. Africa, south of the Sahara) is a cause for serious concern, because the long half-life of the drug may expose reinfecting parasites to subcurative drug concentrations that could select for mefloquine-resistant strains (which are often crossresistant to quinine (17)). The alternative to mefloquine is quinine, given three times a day for 5-7 days. To ensure a high cure rate, quinine is normally given in combination with another drug, usually doxycycline or tetracycline. Apart from the fact that doxycycline and tetracycline cannot be given to children under 8 years or to pregnant women, this regimen has the further disadvantage that patient compliance is very low because of the need to take quinine, which has important side-effects, three times a day. Clindamycin, an alternative to doxycycline that could be used for children and pregnant women, is currently far too expensive to be of use in general health services.

Among the affordable options in malaria-endemic countries, interest is now focusing on artemisinin drugs because of the results of their use over the last 20 years, especially in China, Thailand and Viet Nam (18). These drugs are rapid acting, effective against all strains of $P$. falciparum and $P$. vivax (particularly for the treatment of severe malaria) and are well tolerated.

Owing to the limited amount of research carried out on the artemisinins and concern that their unregulated use could lead to parasite resistance, WHO has until recently recommended that artemisinins should be used only in areas with multidrug-resistant falciparum malaria $(4,15)$. In reality, however, as a result of market pressure they are already widely available, especially in the private sector, in most malaria-endemic countries. In this situation, their uncontrolled use could lead to the appearance of parasite-resistant strains without bringing much benefit to the most vulnerable populations.

So far, resistance to artimisinin drugs has not been documented, although relatively low in vitro parasite susceptibility has been observed in southern China (W.H. Wernsdorfer, personal communication, 1998). Despite neurotoxicity in animal studies at doses that far exceed those used for the treatment of malaria in humans, serious side-effects in humans have not been encountered. The disadvantage 
of these drugs, which is probably related to their short half-life, is that they must be given for at least 5 days for acceptable cure rates to be achieved. For this reason, which is a serious hindrance to compliance, and to reduce the risk of resistance, artemisinins should always be given in combination when used as part of malaria control in endemic countries. Numerous trials have shown that cure rates close or equal to $100 \%$ can be attained by giving the well tolerated combination of an artemisinin drug with mefloquine for 3 days (18). Combinations with other drugs also seem to be effective, but have not yet been studied so widely.

Trials are now taking place to investigate the efficacy and safety of a combination of artesunate and sulfadoxine/pyrimethamine. A fixed combination of artemether with lumefantrine has already been registered, although the company has yet to develop a pricing strategy. Alternative options for combination with artemisinin derivatives include pyronaridine, chlorproguanil/dapsone and atovaquone/ proguanil, although atovaquone/proguanil is still too expensive for endemic countries. If an artemisinin drug with sulfadoxine/ pyrimethamine combination proves safe and effective, it can be recommended as routine first-line treatment in most countries where chloroquine resistance is a problem. Given that sulfadoxine/ pyrimethamine resistance already exists, the useful life of this combination would perhaps be limited, and research on the alternatives mentioned above has high priority. If one of these combinations proves safe and effective, it should be introduced as a replacement for artesunate plus sulfadoxine/pyrimethamine. The replacement would probably have a longer useful life, because there is as yet very little reported resistance to the components. In addition, pyronaridine as well as chlorproguanil/dapsone are better matched kinetically to artesunate than is sulfadoxine/pyrimethamine.

The precondition for such combinations being effective is their correct use. Their large-scale distribution in package form, for example, a blister-pack where the two components are kept together, would improve compliance. A fixed combination formulation would be even better, but this will still take years to develop. The use of artemisinin drug combinations must be undertaken in a responsible way by public health services that can ensure that health staff are trained in their use. In addition, the drugs should not be available without the required information supplied in a locally appropriate form. Correct drug treatment requires diagnosis to be more specific than is currently the case in most places, and the drugs should be free or affordable to the patient populations, so that poverty does not become a cause of underdosing. Finally, the introduction of such combinations should be 
accompanied by monitoring of their impact on mortality and incidence of severe disease (see section 9).

Chloroquine-resistant $P$. vivax is so far not a public health problem because it occurs mainly in areas with intense transmission where most patients are semi-immune, so that chloroquine still has acceptable effectiveness. $P$. vivax cases that fail to respond to chloroquine may be treated with, for example, quinine or mefloquine. In some settings, especially where transmission is not very intense, treatment with primaquine is routinely indicated on clinical grounds to prevent relapse of $P$. vivax infections $(4,14)$.

\subsubsection{Availability and quality of treatment}

Currently, a large proportion of malaria patients in all endemic areas receive some antimalarial treatment without even making contact with formal health services, which are often not present or not functional close enough to the home. The provision of proper disease management, as outlined above, cannot be expected in the absence of formal health services, and it should always be a priority for governments to improve the access of malaria patients to good quality care. Many countries have attempted to provide disease management for malaria, or for a range of locally important disease problems, through community volunteers. Such systems have not always been effective in reducing mortality. However, interest in them is being renewed in malaria control because they offer a possible focal point in a village, linking the population and the health services in:

- the provision of early antimalarial treatment for cases of fever;

- family and community participation in programmes for promoting the use of impregnated mosquito nets;

- the collection of data on indicators of morbidity and mortality.

The number of private medical practitioners and qualified pharmacists is now increasing in some endemic areas, so that their services are of potential importance in malaria control. Although their precise compliance with official treatment guidelines may be difficult to obtain, it is important to provide them with information and education, and enforce regulations aimed at improving the quality of care. They may also be a source of valuable epidemiological information.

For some years, increased attention has rightly been given to treatment that is provided in the home and by informal services that range from authorized pharmacists to market-vendors and shops. Materials have been developed to teach mothers how to recognize malaria symptoms (2). However, as there is evidence that mothers acquire their knowledge on the use of antimalarial drugs where they buy them 
(19), there is currently great interest in developing training, specifically for the people who sell drugs to patients and their carers. This may prove an efficient way of improving patterns of drug use, especially if combined with health education for mothers, other carers and patients. However, there is still a need for field research to examine whether activities directed at drug sellers can, in the long term, lead to more rational use of drugs.

In all circumstances, appropriate training and/or information must be given at the level where drugs are sold and used. Ideally, first-line drugs, and information about them, should be available through the private sector.

With drug policies changing and new treatments becoming available, there is great concern that the drugs available at the periphery are of good quality. Substandard drugs have an impact on the effectiveness and safety of treatment, and parasite drug resistance. It is therefore essential, especially with the introduction of artemisinin derivatives, to strengthen national capabilities for quality control, and over time, also for quality assurance.

\subsection{Management of severe malaria at the periphery}

From a clinical viewpoint, there are good reasons for merging the definitions of severe and complicated malaria, although in some settings, it is useful to have separate reporting of complicated malaria, which can be more rigorously defined. The management of severe malaria in hospital settings is presented in detail elsewhere (20). This section focuses on the management of severe malaria at the periphery.

The priority requirement is the early recognition of the signs and symptoms of severe malaria that should lead to emergency care in an in-patient setting. The signs and symptoms that can be used are nonspecific and are really the signs and symptoms of severe febrile disease (2), which may be severe malaria, another severe febrile disease or concomitant malaria and severe bacterial infection (K. Marsh, personal communication, 1998).

The signs and symptoms of severe febrile disease in children are a history of fever, plus at least one of the following:

- prostration (inability to sit), altered consciousness, lethargy or coma

— breathing difficulties

- severe anaemia

- convulsions 
— inability to drink

- persistent vomiting.

For adults, the same signs and symptoms are valid, with the addition of:

- dark and/or limited production of urine.

Patients with prostration and/or breathing difficulties should, if at all possible, be treated with parenteral ${ }^{1}$ antimalarials and antibiotics. If the clinical condition permits, other patients may be treated with oral antimalarials that must be fully effective.

For settings where patients with severe malaria present regularly and it is not possible to refer them rapidly to facilities able to treat severe malaria, a basic package for initial treatment could be made available, supported by training and supervision. There are initial encouraging results from Malawi on the provision of such a package. The training should emphasize that, if at all possible, patients who have improved after initial treatment, but are still ill, should be referred.

The contents of the package may vary according to the location, but the minimum should include:

- a parenteral antimalarial

- a parenteral antibiotic

- an effective oral antimalarial

- a rectal anticonvulsant.

The parenteral antimalarial drug should be quinine, an artemisinin derivative or sulfadoxine/pyrimethamine, if the parasite is known to be susceptible to the latter combination.

Rectal formulations of artesunate are now more available and are likely to be chosen for pre-referral treatment of severe malaria. However, attention should be paid to the need for a complete curative treatment.

It may be necessary, especially if quinine is used, to provide glucose intravenously or via a nasogastric tube. However, there are serious doubts about whether liquids can be given safely by either of these routes outside hospital. This issue should be examined by operational research under local conditions.

A rectal anticonvulsant, for example, diazepam, is to be used in the case of convulsions. Recent research indicates that routine administration of phenobarbital may increase mortality in children with severe malaria (K. Marsh, personal communication, 1998).

\footnotetext{
${ }^{1}$ Normally, parenteral administration will be intramuscular.
} 


\section{Drug resistance of malaria parasites}

\subsection{Monitoring of drug resistance}

The primary objective of monitoring drug resistance is to evaluate the efficacy of the recommended treatment options for malaria at the local level, with particular emphasis on falciparum malaria. The most suitable method to achieve this objective is monitoring the therapeutic efficacy of antimalarial drugs (21), which has been recently modified $^{1}$ by WHO to replace the standard in vivo test. Other levels of monitoring exist, including in vitro susceptibility testing of parasites and the study of genetic markers of resistance, both of which are very useful for specific research purposes (see sections 6.1.2 and 6.1.3).

\subsubsection{Monitoring of therapeutic efficacy}

In 1994, WHO set up a new system for monitoring the therapeutic efficacy of antimalarial drugs used for the treatment of uncomplicated falciparum malaria, based on clinical evaluation of selected malaria patients, using a limited number of follow-up examinations (15). A detailed protocol was developed in 1996 for areas with intense transmission, specifically for children under 5 years (21). The basic test consists of recording essential patient information: clinical assessment, body temperature, body weight, parasitaemia and haemoglobin levels on day 0 (prior to treatment), and providing supervised treatment. Clinical and parasitological examinations are repeated on days 3,7 and 14, and haemoglobin levels of anaemic children only are reevaluated on day 14. Clinical and parasitological criteria are used to classify the response to treatment as adequate clinical response, early treatment failure (from days 1 to 3 ) and late treatment failure (from days 4 to 14). Through a series of interregional and intercountry workshops organized by WHO, ${ }^{1}$ the basic protocol has been specifically adapted for areas with low or moderate transmission. The modifications include enrolment of patients of all age groups, treatment based on reappearance of parasitaemia and follow-up of patients up to day 28.

As the monitoring of therapeutic efficacy is required for routine evaluation of drug efficacy, it should be incorporated into the plan of action of all malaria control programmes. Because of changing patterns of drug resistance, the efficacy of the available treatment options should be assessed at regular intervals (e.g. every 2 years) or

Assessment of therapeutic efficacy of antimalarial drugs for uncomplicated falciparum malaria. Geneva, World Health Organization, 1997 (draft document 29.2.97; available on request from the Roll Back Malaria project, Communicable Diseases, World Health

Organization, 1211 Geneva 27, Switzerland). 
whenever there are increasing numbers of clinical reports of drug failures. The proportion of early and late treatment failures after supervised treatment should help policy-makers decide whether to update the malaria treatment guidelines.

Monitoring of therapeutic efficacy is complex and requires specific technical expertise. Given the present requirements of the test, general health service staff with minimal supervision are unable to carry it out. However, the protocol can be used to train staff of the national malaria control programmes in operational research methods. The core protocol can be used in field trials of antimalarial drugs or drug combinations, and whenever possible, the basic observations should be incorporated into new clinical trials, so that the results can be used to monitor therapeutic efficacy.

The most important requirements for effective monitoring of therapeutic efficacy are the selection of a minimum number of representative sites, and maintenance of quality in the performance of the study. The evidence available does not allow simplification of the method, and further research on early predictors of treatment failure is needed to develop a test of shorter duration. The current test, however, can provide the basis for further research and investigators can add other observations (e.g. day 5, day 11 or day 28 follow-up examinations) to the basic test to address specific issues. The long-term goal, however, remains to develop procedures which can be incorporated into routine patient management, permitting continuous monitoring of drug response.

Interpretation of the clinical response will be determined mainly by the objective of malaria treatment (i.e. clinical or radical cure), instead of endemicity levels (15). In Africa, where clinical cure is the objective of treatment, a follow-up of 14 days has been adopted for all age groups in areas of low or moderate transmission, and only for children under 5 years in areas of intense transmission. In countries where treatment aims at radical cure, a minimal duration of 14 days is recommended for the test, if follow-up at 21 and 28 days is not feasible.

Although $P$. vivax resistance to chloroquine has been reported in a few countries, this has so far had a limited effect on the clinical management of patients with vivax malaria. The therapeutic efficacy of chloroquine against $P$. vivax could be monitored using a protocol developed for $P$. falciparum.

With more experience and research, the methodology for monitoring drug resistance should be refined on a regular basis. Of specific inter- 
est will be research to determine the significance of early and late treatment failures, in terms of the risk of rapid progression of the disease to severe malaria and death; persistent morbidity; and the burden on the health facilities resulting from recurrent unsuccessful treatment.

\subsubsection{In vitro susceptibility testing}

Testing parasite susceptibility to antimalarial drugs in vitro is not routinely required by all malaria control programmes. It is, however, a very useful tool to investigate specific issues, such as:

- temporal and geographical trends in parasite susceptibility, which may be an indication of future changes of drug efficacy in vivo;

- patterns of cross-resistance of $P$. falciparum to different drugs;

- assessment of the parasite baseline susceptibility to new drugs, before their use in a certain area.

In order to understand the associated dynamics of resistance, in vitro susceptibility testing of artemisinins should be encouraged in areas where these drugs are being introduced. In vitro testing of mefloquine and quinine may serve as an early warning system in areas of low or incipient resistance, since in such areas, long follow-up periods may be needed to detect low levels of treatment failures with these drugs.

\subsubsection{Surveillance-based molecular markers of drug resistance}

Research in this area is very active. $P$. falciparum resistance to pyrimethamine and proguanil is associated with point mutations in the $d h f r$ gene, and sulfadoxine resistance is linked to point mutations in dhps. However, molecular surveillance of resistance to sulfadoxine/ pyrimethamine still requires the identification of a few genetic markers (specific combinations of $d h f r$ and $d h p s$ gene mutations) that are highly predictive of treatment failure. The precise molecular mechanism of resistance to chloroquine and other antimalarial drugs, such as mefloquine, halofantrine and quinine, is not clear.

The validation of the genetic markers will require comparative pre- and post-treatment observations on the diversity of parasite isolates (to exclude reinfections), in vitro susceptibility testing, assessment of clinical response and measurement of drug levels in the blood. Results of studies coordinated by WHO will be available in the year 2000, and should help consolidate and improve the current methods for monitoring drug resistance. 


\subsection{International monitoring of the drug response of P. falciparum}

The main objectives of international monitoring of drug resistance are to analyse the dynamics of drug resistance and share the information among countries. As part of the analysis, the international monitoring may also serve to check and evaluate drug utilization strategies or other malaria control activities aimed at reducing or preventing the selection of parasite resistance.

A limited number of centres need to be established in Africa, south of the Sahara, and elsewhere as repositories of parasite isolates (from blood samples, fingerpricks taken at the time of clinical failure and blood smears) for studies on drug resistance, malaria genetics, immunology and pharmacology.

The capability for pharmacokinetic studies should be strengthened at the regional level. Selected WHO Collaborating Centres should be used for such studies and for training purposes.

\subsection{Antimalarial treatment policy}

The level of treatment failures, which serves as a basis for the replacement of first-line treatment, varies and depends on the drug options available and the financial, institutional and personnel resources in the country. It has been suggested that malaria treatment guidelines be updated when treatment fails in $25 \%$ of cases (counting both early and late treatment failures), but it is widely recognized that no uniform efficacy threshold can be established.

The cost of sulfadoxine/pyrimethamine has been considerably reduced during recent years to that of chloroquine, and the change from chloroquine to sulfadoxine/pyrimethamine can be made earlier than originally thought. Apart from chloroquine and sulfadoxine/ pyrimethamine, it is likely that the decision whether to change antimalarial drugs will be dependent not only on the level of treatment failures, but also on cost and other operational factors $(4,15)$.

At present, many national malaria control programmes are unable to implement several different malaria treatment guidelines. With the steady improvement in the surveillance of drug resistance on the one hand and the introduction of more expensive and toxic drugs on the other, it may be necessary to develop and implement regional treatment guidelines.

Greater efforts must be made by national governments and health programmes to ensure that populations at risk have easy access to appropriate and affordable first-line antimalarial drugs, as close as possible to the community. In areas or situations where the parasite is 
resistant to the first-line treatment, effective second-line treatment must always be available.

The development of appropriate legislation is important to improve drug registration and the use of drugs (especially in the informal sector). Such legislation will also help establish effective mechanisms for drug quality control. WHO should strengthen capacity for quality control of antimalarial drugs on a regional basis.

\subsection{Containment of parasite drug resistance}

A direct relationship between malaria transmission and parasite drug resistance has not been clearly established. However, a recent review paper (22) suggests that interventions that reduce transmission may also lead to decreased drug resistance in $P$. falciparum, as was demonstrated in north-east India (23). In reality, it is not yet known what effect inadequate vector control has on the kinetics of the spread of parasite drug resistance, nor the effect specific interventions (e.g. the introduction of insecticide-treated bednets) have on the containment of parasite drug resistance in areas of intense transmission in Africa, south of the Sahara.

$P$. falciparum multidrug resistance is the major threat to malaria control in many countries of south-east Asia. In the affected areas, conventional vector control methods of indoor spraying are not applicable and there is an urgent need to use alternative methods (including personal protection, repellents or causal prophylaxis) to reduce the risk of transmission of drug resistance.

Some data have demonstrated an association between malaria epidemics and parasite drug resistance. Since mass drug administration - one of the major interventions in epidemic control - may contribute to increased drug resistance, it should always be followed by an intensive follow-up procedure that includes, in addition to vector control, active case detection and radical treatment to eliminate residual foci of drug resistance.

Appropriate combination therapy may help to prevent or contain drug resistance provided that the mechanisms of drug resistance to the individual drugs are independent, and the components show complementary pharmacokinetic actions (24).

Where intensive population movements occur into areas with $P$. falciparum multidrug resistance, there is a need to protect people who migrate into these areas - using appropriate strategies (e.g. personal protection and standby treatment) - and to screen and treat those who return from problem areas. Causal prophylaxis with daily primaquine could be considered for high-risk groups staying temporarily 
in areas with $P$. falciparum multidrug resistance, after prior screening for glucose-6-phosphate dehydrogenase deficiency (25).

\section{Malaria epidemics: prediction, preparedness and control}

Malaria epidemics continue to threaten large areas of the world. These areas, in tropical and subtropical regions, have generally been the sites of the major regional epidemics of the past. Each epidemic arises from the sudden exposure of non-immune individuals to intense disease transmission. The extent of malaria transmission is greatly influenced by ecological and social conditions, particularly hydrological and meteorological abnormalities, population movements and other factors.

The impact of malaria epidemics extends beyond the increase of specific morbidity to the general health of the affected population which is often already suffering from the effects of crop failure, economic crisis, war or civil disturbances. Therefore, individuals in epidemic-prone areas are generally not only physically weak, but also exposed to other diseases and unable to obtain appropriate care.

Malaria epidemics may be the result of:

- Major changes (e.g. environmental) in the eco-epidemiological system, pushing the area towards a new equilibrium of higher endemicity. In the absence of intervention, high endemicity will be established and remain.

- Premature termination or unplanned interruption of antimalarial measures which had previously controlled areas with all the epidemiological characteristics of high endemicity. These epidemics are true resurgences, arising from failures of control. Left alone, the original endemic situation will be re-established in a few epidemic seasons.

\subsection{Epidemic risk and epidemic-prone areas}

Epidemics occur in areas or situations where most of the conditions for intense malaria transmission exist, but normally one or more essential factor is lacking or is insufficient. Thus in normal years, malaria incidence is low and the transmission season short, and consequently the population is mostly non-immune. In years when the usually weak factors are exceptionally prominent and/or prolonged, the resulting intense transmission produces an epidemic. The epidemics often occur in identifiable epidemic-prone areas, where they exhibit a certain periodicity (often a 2-7-year cycle), or are linked to ecological and social disturbances. The areas along the edges of 
malaria endemicity often show this periodicity and can also be considered as epidemic prone, whether they are the fringes of deserts or the upper limits of highlands.

Epidemic-prone areas can be classified, according to the main factors responsible for triggering an epidemic, into:

- Endemic areas subject to a sudden increase in the number of exposed non-immune individuals, caused by:

- the arrival en bloc of non-immune populations (e.g. refugees or displaced populations) into a malarious area;

- the mixture of large numbers of immune and non-immune people living in primitive conditions (e.g. in temporary labour camps and development project sites).

- Hypo- or meso-endemic areas subject to a sudden increase in vectorial capacity, caused by:

- an abrupt rise in Anopheles density due to abnormally heavy rains, and/or increased survival of the mosquitos due to prolonged warm and humid weather;

- acceleration of the parasite sporogonic cycle due to exceptionally long and warm summers;

- invasion of a more efficient vector into areas where local vectors were not able to maintain intense transmission, or areas where no vector previously existed.

- Hypo- or meso-endemic areas subject to environmental modifications which may lead to both increased vector density and human population movement, such as:

- agricultural development;

- rapid, uncontrolled growth of cities in tropical areas.

- Previously endemic areas, which fail to maintain previously effective controls because of:

- resurgence of malaria transmission (leading to post-eradication epidemics);

- progressive spread of parasite chloroquine resistance, particularly in Africa, south of the Sahara, during the past two decades. While in some situations, parasite drug resistance has assumed epidemic proportions, epidemics often act as a major vehicle for the spread of drug-resistant strains.

\subsection{Epidemic preparedness, prediction and prevention of epidemics}

When an epidemic occurs, the urgency for action seldom allows enough time for planning and implementing the necessary control measures, unless there is an adequate degree of preparedness. In 
epidemic-prone areas, this preparedness should come from an appropriate prediction system based on monitoring epidemic risk factors.

Epidemic and emergency preparedness is being promoted as an essential activity of health services. In line with this, WHO is supporting the organization and work of intercountry emergency preparedness teams in Africa which will collaborate with affected countries. In epidemic-prone areas, it is essential that a close collaboration is established between the specialized antimalarial services and the emergency preparedness teams. The specialized services could then assist in the:

- identification of epidemic-prone areas, the main risk factors and alarm signals;

- monitoring of risk factors;

- planning, implementation and evaluation of preventive or control measures, taking into account essential epidemiological characteristics (e.g. parasite sensitivity to drugs and vector susceptibility to insecticides).

The concept of risk detection should be considered in relation to the time available for implementing an appropriate response. After recognizing an imminent epidemic risk, it is particularly important to be able to estimate the potential magnitude of the forthcoming epidemic wave, as well as the area of potential spread.

\subsection{Epidemiological information systems}

Information systems have often been designed to provide managerial and epidemiological data thought necessary to monitor the impact of interventions and the implementation of control programme activities.

Renewed interest in malaria epidemics has made malariologists and epidemiologists aware of the fact that most epidemics are caused or greatly influenced by meteorological or socioeconomic determinants. However, most antimalarial services have still not set up mechanisms to monitor these variables. It is essential that health services have the epidemiological competence to select suitable indicators and the capacity to coordinate intersectoral collaboration to monitor them in time.

An essential component of any epidemiological information system is an appropriate geographical information system. As epidemics are catastrophic events, their study requires precise definition of time and location. For both the implementation of control interventions and understanding of risk dynamics, it is necessary to define the geographical limits of each epidemic as much as possible. 
As most malaria epidemics are the result of abnormal meteorological conditions, climate monitoring will provide the most useful indicator of epidemic risk. Analysis of past records will permit the identification of alarm signals (e.g. early and prolonged rains, floods and monsoon failures), which could alert the epidemic preparedness system to prepare an adequate response.

Information from meteorological satellites, particularly the normalized difference vegetation index (NDVI) ${ }^{1}$ may be very useful in the identification, confirmation and demarcation of epidemic-prone areas. The usefulness of satellite data as risk indicators is limited, as rain monitoring provides an earlier warning. Consideration should nevertheless be given to the potential usefulness of other indicators obtained by satellite, such as cold cloud duration (CCD), if they can be obtained earlier than currently possible.

Monitoring the relevant meteorological variables remains the most useful indicator in areas where the risk of epidemics is associated with:

- drought, such as the failure of the south-west monsoon in the malarious intermediate zones of Sri Lanka;

- abnormally high temperature, such as in the highlands (around $2000 \mathrm{~m}$ ) of east Africa.

Epidemics resulting from environmental modifications or social disturbances will require the continued alertness of malaria epidemiologists to the social and economic conditions in malarious or potentially malarious areas.

Early detection of epidemic situations requires a definition of normality. The most practical method is the determination of epidemic indices by plotting the median and the third quartile of the malaria incidence (or merely the numbers of malaria cases) every calendar month of previous years. This defines a normal range on which to plot the current data in order to detect an abnormal increase. Malaria programmes should support district medical officers to ensure that at least a few health facilities (hospitals and health centres) in epidemicprone areas adopt this method of epidemiological analysis.

\subsection{Epidemic response}

When it is not possible to prepare in advance for an epidemic, the recognition of an alarm signal of an impending epidemic should be

Used as a proxy for monitoring growth and types of and changes in seasonal vegetation.

Used as a proxy estimate for rainfall. 
followed by the implementation of measures, which may consist of the following:

- transmission prevention measures (e.g. indoor residual spraying) before the start of the epidemic, if resources are available;

- if such measures cannot be implemented in time, it is necessary to ensure that there are adequate stocks of antimalarial drugs, several fold above normal levels of consumption, and strengthen the systems for early detection of emerging epidemics, in as many as possible of the health-care facilities in the area at risk.

The response to a reported epidemic, from whatever source (health services, private health providers, political authorities or the press) should include:

1. Confirmation of diagnosis (parasite species, if possible); recording the severity, duration of illness, occurrence of deaths, and consistency of case definitions. If malaria is confirmed, the parasite species involved and transmission dynamics should be documented.

2. Demarcation of the affected area.

3. Assessment of the local capacity (health services, community participation and intersectoral collaboration) to manage the situation and of the need for assistance from within the country and perhaps from international collaborators.

4. Strengthening diagnostic and treatment facilities within the health services and in whatever informal systems can be mobilized (e.g. local authorities and organizations, schoolteachers and home drug suppliers).

5. Planning and organizing emergency control measures, possibly implementing selected interventions in time to influence the course of the epidemic.

It should be emphasized that, ideally, the work described in points 3-5 should be carried out well before the outbreak of an epidemic.

The main objectives of epidemic control should be to:

- provide adequate relief to the affected population;

- contain transmission, if possible, in the affected areas;

- prevent further spread of the epidemic;

- improve emergency preparedness in order to prevent future epidemics.

Emergency control may include mass drug administration or, preferably, mass treatment of fever, which normally includes treatment of every patient complaining of current or recent fever, and everyone in the patient's household. These measures are mostly indicated in the 
case of refugees or displaced populations before a health-care system can be organized in the camps. The drug selected for mass treatment should have recognized effectiveness, but its use should not threaten the supplies needed for the treatment of actual cases. The objective of mass drug administration is to reduce rapidly the parasite reservoir during the period of intense transmission. Primaquine, if available, should be added to the drug regimen for its gametocytocidal effect. All efforts should be made to control transmission (e.g. by space spraying and indoor residual spraying) whenever mass drug administration is implemented, as mass treatment of a non-immune population at a time of very intense transmission will exert a high selection pressure for parasite drug resistance.

\subsection{Post-epidemic action}

Any epidemic should be considered as an opportunity to improve the epidemiological and health services in epidemic preparedness, and the collaboration between these services and the malaria control programme.

It is essential to determine:

- what deficiencies prevented the prediction of the epidemic or hindered the implementation of preventive measures;

- what problems, if any, affected the early detection, confirmation of an epidemic or timely response;

- what indicators should be monitored in order to improve the detection of epidemic risk.

It is also vital to strengthen case management and transmission control capabilities in order to improve risk detection and prevention of further epidemics in subsequent transmission seasons in the same and similar areas.

\section{Prevention of malaria}

The Global Malaria Control Strategy stresses the selective use of preventive measures wherever they can lead to sustainable results. Such measures should be aimed at halting the deterioration of the malaria situation, minimizing the wasteful use of resources and contributing appropriately to the development of health services, intersectoral cooperation and community participation.

\subsection{Selective vector control}

Selective vector control involves the targeted use of different vector control methods alone or in combination to prevent or reduce 
human-vector contact cost-effectively, while addressing sustainability issues. Implementation of control measures depends on a number of factors, such as malaria epidemiology, the available information, the potential and limitations of each method, infrastructure and institutional capacities. These vary within and among countries and regions.

\subsubsection{Indoor residual spraying}

Non-selective coverage, as used for DDT and other insecticides in the past, is no longer a recommended strategy. Reduction of widespread coverage is still needed in the Americas, Asia and in some areas of Africa (where malaria transmission is focal and unstable, and in epidemic-prone areas). Given the financial and human resources involved, combined with the potential for vector resistance and environmental concerns, indoor residual spraying should be used only in well defined, high or special risk situations. DDT is being phased out because of its previous widespread use in the environment, and the resulting political and economic pressure.

Epidemiological indicators used to decide whether to implement indoor residual spraying should be revised to take into account transmission patterns, which may vary over time and area. Major sites considered for spraying could be further delineated to the smallest possible operational units, with well defined targets for spraying. Criteria for deciding whether to start or stop spraying operations are also needed.

A still finer analysis of epidemiological information allows spraying to be targeted to houses that are in places where the risk of transmission is highest, such as those near major breeding sites. Depending on the preferred resting sites of the vectors, it is even possible to restrict spraying to certain surfaces of houses.

\subsubsection{Use of DDT in malaria control}

The use of DDT was addressed at the 1995 meeting of the WHO Study Group on Vector Control for Malaria and Other MosquitoBorne Diseases (26). The Study Group stated that DDT may be used for vector control, provided that it is only used for indoor spraying, it is effective, the WHO product specifications are met, and the necessary safety precautions are applied for its use and disposal. The Study Group's conclusions are reproduced with minor editorial changes in Annex 1. The Expert Committee re-examined these conclusions and endorsed them as still valid.

In some countries, where the local malaria vector is still susceptible, DDT is used for indoor residual spraying. However, in almost all 
countries, DDT has been banned for use in agriculture and a number of countries have extended this ban to public health applications. Several other countries (e.g. India, Mexico and South Africa) are considering or have decided to phase out DDT use in their public health services over periods of between 3 and 8 years.

In accordance with their mandate, previous meetings of the relevant WHO Expert Committees and Study Groups have focused mainly on the human toxicological aspects of DDT and other insecticides. The targeted application of insecticides to indoor walls in order to interrupt disease transmission greatly reduces dispersion of the chemicals into the environment. For this reason, the environmental risks from such targeted measures were considered minimal, certainly when compared to those associated with agricultural applications, in which the quantities of insecticides released are much greater than for public health use. Environmental protection agencies argue, however, that significant amounts of DDT intended for public health use still end up in the agriculture sector through illicit trading practices, and therefore in the environment. There are insufficient data available for exact quantification, but the significance of this is thought to be minimal. It is clear, however, that in the broader context of sustainable development and in line with the principles agreed at the United Nations Conference on Environment and Development (27), WHO's concern now goes beyond human toxicology to the impact of DDT use on the earth's resources and biodiversity.

\subsubsection{Use of insecticide-treated materials}

Insecticide-treated materials, such as eaves-strips, curtains, hammock nets, raffia foldable shields and fly curtains, have been used as alternatives to mosquito nets in some settings. This approach will expand the acceptability and flexibility of impregnated materials.

The safety of pyrethroid-treated hammocks in jungle areas, especially in the Amazon region and south and south-east Asia, needs to be investigated. Information on the safety of using treated cloth in, for example, some army uniforms, may be available.

In endemic areas, insecticide-treated materials should, in the long term, become normal household goods that can be bought from appropriate outlets, such as pharmacies and local shops. However, mechanisms to meet the needs of the poor must be urgently explored.

A challenge for the control programme regarding impregnated materials is the provision of short-term donations from interested parties in the commercial sector which may have a negative impact in the 
long term. Therefore "donors" should be encouraged to contribute to more sustainable programmes.

To improve the affordability of mosquito nets and insecticides, appropriate measures (e.g. tax exemption, price control and exceptional inclusion of both the nets and insecticides in a list of essential drugs) need to be considered. Social marketing is regarded as an important initiative to create demand that will progressively be supplied by the commercial sector. However, the public sector should ensure quality assurance for netting materials and insecticides. Simple tests for monitoring the presence of insecticides on nets need to be developed as a matter of urgency.

Re-treatment is a critical component of sustainable programmes for promoting the use of insecticide-treated materials. Therefore, promotional activities to increase people's awareness of the need to re-treat materials should be intensified. Several options for re-treatment are available, including providing individual doses (in the form of sachets or tablets), which are thought to make insecticides more affordable at the peripheral level. However, careful monitoring of re-treatment of materials is important for detecting both inadequate and excessive doses of insecticides. Methods to improve the wash-resistance of insecticide deposit, such as by permanent impregnation, also need to be further investigated.

The impact of insecticide-treated nets on overall mortality has been clearly established. However, there is a need to monitor their longterm effect on the immunity of the human population and the resistance and behaviour of the mosquito population. Admission of patients to hospital with anaemia or severe malaria may serve as a rough indicator of trends in morbidity and mortality related to malaria control activities, including the use of impregnated materials. Monitoring and evaluation of the use of insecticide-treated materials should be integrated into all malaria control programmes.

\subsubsection{Management of malaria in development projects}

Development projects often inadvertently contribute to the risk of malaria. Therefore, policies and legislation need to be developed and enforced to prevent this additional risk, and to counter the detrimental effects of the projects on the malaria situation.

Health-impact assessments are being promoted as an essential component of environmental-impact assessments for important development projects. Health-impact assessments should include a full evaluation of the effects of the project on the epidemiology of 
malaria, not only in the immediate vicinity of the project or on those involved in it, but also in the whole area influenced by the project.

If such assessment is properly conducted at the planning stage, a relatively minor investment, properly guided, will not only result in the prevention of man-made malaria, but could also contribute to better control of malaria in all the areas influenced by the project. In contrast, failure to conduct an assessment at the planning stage will frequently result in malaria epidemics or resurgence of the disease with high morbidity and mortality, and will require major investments to control the situation.

These principles are now widely accepted, but are not always put into practice during the planning phase for financial or other reasons. Management of malaria during development projects requires intersectoral collaboration, and provides an opportunity to establish links between workers in different disciplines that could be of value in other malaria control programmes not related to the development project.

Current examples of environmental management of malaria through vector control also need to be documented.

\subsubsection{New developments in Africa}

There is increased knowledge of the heterogeneity of malaria transmission patterns in Africa, south of the Sahara: transmission patterns reflect changes in human-related factors (e.g. urbanization of the population (at present $40 \%$ ), infrastructure and social development). Urbanization has raised awareness of the need for malaria control, and political commitment to support this control has simultaneously increased. The urban environment has offered better opportunities for selective vector control.

The following developments have been made in rural areas:

- Where malaria transmission is stable, the use of insecticidetreated materials is the preventive method of choice when used correctly.

- Where transmission is unstable, indoor residual spraying may be appropriate, provided the infrastructure to maintain the programme is available. In recent years, indoor residual spraying campaigns have become increasingly selective, partly because of the implementation of the Global Malaria Control Strategy.

- In a few areas where both impregnated materials and indoor spraying would be feasible, the desirability, cost-effectiveness and possible synergy of both methods are being explored. 
In epidemic-prone areas and areas with a very short malaria transmission season, indoor spraying is more suitable than using impregnated materials to contain epidemics.

\subsubsection{Use of gametocytocidal drugs}

The fact that artemisinins reduce gametocyte counts in infected patients and probably also reduce transmission suggests that, under conditions of low transmission, treatment of malaria patients with these drugs may have a significant role in the prevention of malaria (28).

\subsection{Chemoprophylaxis}

The available drugs for chemoprophylaxis are limited to chloroquine, proguanil, pyrimethamine/dapsone, mefloquine and doxycycline. Increasing parasite drug resistance and drug-specific side-effects make rational guidelines for chemoprophylaxis more and more difficult to compile. There is no ideal drug for prophylaxis. Chemoprophylaxis is limited to travellers, special groups (e.g. armies) and, in certain situations, pregnant women.

\subsubsection{Chemoprophylaxis among non-immune people visiting endemic areas}

In recent years, there have been improvements in providing advice on chemoprophylaxis in areas where it is required, and in taking into account the type of traveller and the specific risks they face. In some areas, possible side-effects of the recommended antimalarial may outweigh the risk of acquiring a malaria infection. In areas with vivax malaria, the use of chloroquine prophylaxis may need to be reevaluated. All travellers need to take adequate precautions to prevent mosquito bites.

Travellers should always consult their doctor to discuss specific contraindications of antimalarial drugs well before travel. The standardization of national and international guidelines with those of WHO recommendations (29) is desirable, since differences between them may lead to confusion, and travellers may not take any prophylaxis at all.

Factors such as patient compliance and drug safety that complicate long-term prophylaxis need more attention and research (4).

\subsubsection{Prevention of malaria in pregnancy}

Pregnant women in malaria-endemic areas all too often do not receive the preventive and curative care needed, contributing to the 
avoidable and unacceptably high maternal and infant mortality. Health care for these women must be a comprehensive "package", based on the extent of the problem and opportunities for intervention. In areas of high malaria transmission, this package should include prevention of malaria infection through health promotion and intervention, as well as methods to reduce the consequences of infection. This will include the use of antimalarials as prophylaxis or as intermittent treatment, possibly the use of insecticide-treated mosquito nets, and access to early diagnosis and effective treatment for anaemia and clinical malaria.

In endemic areas, effective prevention of malaria in pregnancy decreases the incidence of low birth weight and severe maternal anaemia. Weekly chemoprophylaxis used to be the method of choice, but chemoprophylaxis is increasingly hampered by parasite drug resistance, contraindications of certain drugs and poor patient compliance. In the search for alternative strategies, "intermittent" therapy has been proposed as a simple and cost-effective method in highly malarious areas. Intermittent therapy involves the administration of full, curative-treatment doses of an effective antimalarial drug at predefined intervals during pregnancy.

In areas of east Africa with increasing chloroquine resistance, largescale trials have shown that intermittent treatment with a singletreatment dose of sulfadoxine/pyrimethamine at the beginning of the second and third trimesters significantly reduces the prevalence of anaemia and low birth weight - the latter being the greatest single risk factor for infant mortality. Studies in Kenya and Malawi indicate that rates of placental malaria, severe anaemia and low birth weight can be significantly reduced if women in their first and second pregnancies receive intermittent sulfadoxine/pyrimethamine as part of antenatal care. Furthermore, the drug was well tolerated by pregnant women, and adverse reactions were uncommon (30-33, A. Machesa, personal communication, 1997).

In Malawi, intermittent sulfadoxine/pyrimethamine was successfully introduced in 1993 as national policy for the prevention of malaria in pregnancy, resulting in a decrease in the incidence of low birth weight in women in their first pregnancy (34). It can be concluded that in areas where there is a high risk of $P$. falciparum infection in pregnancy, where $P$. falciparum is susceptible to sulfadoxine/pyrimethamine and where the treatment regimen can be administered correctly, intermittent treatment with sulfadoxine/pyrimethamine is safe and effective for reducing the consequences of malaria in pregnancy. In malaria-endemic areas, comparing the birth weights of 
first-born infants with those of infants born to mothers who have had several pregnancies may be a suitable indicator of the efficacy of malaria control in pregnancy. This ratio of birth weights may be used to identify the endemic areas where malaria control in pregnancy is inadequate and where intermittent treatment is beneficial to pregnant women (35).

Further studies in Kenya and Malawi demonstrate reduced efficacy of intermittent sulfadoxine/pyrimethamine treatment in HIV-infected pregnant women, indicating that these women may require more frequent intermittent treatments $(30,36)$. There are indications that HIV infection may interfere with the maintenance of pregnancyspecific immunity acquired during first and second pregnancies, placing HIV-infected women who have had several pregnancies at risk of the severe consequences of malaria in pregnancy (37).

\subsection{Malaria vaccines and basic research}

The Expert Committee welcomes the investment of basic research in encouraging the resurgence of interest in the development of new tools for malaria prevention and control. Vaccines and, for example, transgenic mosquitos offer exciting possibilities in the context of an integrated malaria control programme, if and when they become operationally available.

\subsection{Trends in insecticide resistance}

The resistance of malaria vectors to insecticides has resulted far more from the use of insecticides for agricultural purposes than from vector control operations for public health.

Widespread dieldrin resistance developed in the 1960s, and is still present in many anopheline populations. Although the use of dieldrin was abandoned long ago, the mosquito resistance mechanism confers some cross-resistance to phenyl-pyrazoles, a newly developed class of insecticides.

Unlike dieldrin resistance, DDT resistance in malaria vectors took longer to develop and was not so widespread. It was generally induced by a DDT-specific resistance mechanism (glutathione $S$-transferases). However, in west Africa, another resistance mechanism, $k d r$ (knock down resistance), has been recently found which confers crossresistance to DDT and a wide range of pyrethroids; it results from a point mutation in sodium channels - the target site for DDT and pyrethroids. Polymerase chain reaction diagnostic tests can be used to detect $k d r$ in members of the An. gambiae complex. The $k d r$ gene was maintained or further selected in mosquito populations by intensive 
use of pyrethroids in agriculture and household pest control. The gene can be maintained at a low frequency in mosquito populations over decades. Pyrethroid resistance has already been found in some important malaria vectors such as An. gambiae s.s. in west Africa, An. albimanus in Central America, An. sacharovi in Turkey and An. stephensi in India, Pakistan and parts of the Arabian Peninsula.

The impact of $k d r$ on the behaviour of An. gambiae s.s. and the efficacy of pyrethroid-treated nets has been recently investigated using experimental huts in two countries of west Africa (Benin and Côte d'Ivoire). For each country, a comparison was made between areas where mosquitos were resistant and where they were susceptible. Although the allelic frequency of $k d r$ was very high (more than $80 \%$ ) in areas of resistance in both countries, permethrin- and deltamethrin-treated nets still offered protection against mosquitos: the repellent and killing effects, as well as reductions in blood feeding, were maintained at similar levels in both resistant and susceptible areas. The impact of $k d r$ is to be further evaluated at the operational level when nets are used for community protection - mass killing of the mosquito population is expected. It will be also important to assess the impact of pyrethroid resistance mechanisms other than $k d r$, such as esterases or oxidases. So far, $k d r$ has only been found in the savanna form of An. gambiae s.s. and in west Africa, but its presence has also been strongly suspected in An. stephensi, An. albimanus and An. sacharovi.

In areas where $k d r$ is currently absent, it is unlikely to be selected by the use of insecticide-treated materials alone. However, other mechanisms may also be involved in pyrethroid resistance, some of which are already found in malaria vectors. Considering the increasing use of pyrethroids in agriculture and in urban areas, the development of pyrethroid resistance in malaria vectors should be regarded as a very important issue.

Networks for monitoring insecticide resistance in malaria vectors have been recently launched in Africa. In addition to classical bioassays, the resistance mechanisms involved will be identified and their impact on the efficacy of impregnated materials evaluated. Similar networks should also be established in other areas where vector control is envisaged.

Although considerable hope is now centred on the use of insecticidetreated materials, its success relies almost entirely on pyrethroids, which are the only insecticides currently available for this purpose. Therefore, a high priority must be given to the search for nonpyrethroid insecticides for treating materials. The search for effective 
and practical strategies for management of insecticide resistance in mosquitos should be encouraged. Every effort should be made to prolong the useful life of available insecticides and to develop alternative preventive measures.

\subsection{Cost-effectiveness of preventive measures}

Too little is known about the true cost of malaria control and the costeffectiveness of different interventions in different epidemiological circumstances. Such issues should be addressed: every control programme should be evaluated, costed and compared with similar programmes in different epidemiological conditions.

\section{Information systems and operational research}

\subsection{Epidemiological indicators}

Accurate epidemiological information is critical for assessing the extent of public health problems, and for planning and evaluating disease control programmes. While such information is collected primarily for the purpose of guiding local and national control efforts, it is also collated and used by international organizations for assessing regional and global trends. For this reason, agreement on standardized approaches to data collection and reporting is extremely important.

The wide variety of malaria data collected and reported by different malaria control programmes makes comparisons between countries extremely difficult. Since the final decision about the type and frequency of data collection should be made at the level at which the data will be analysed and used, no single set of data will fit all epidemiological situations and countries. However, agreement on standard definitions of malaria morbidity and mortality, and on a limited number of "indicators" that could be used in all situations to monitor malaria control activities, would represent a major step forward. The use of such core indicators would not preclude countries from collecting other information they consider necessary to monitor the progress of their individual plans of action for malaria control.

\subsubsection{Standardized case definitions}

Morbidity and mortality

Definitions of malaria morbidity and mortality will vary, depending on diagnostic capabilities at different levels of the health-care system. Whenever possible, malaria case data should be reported by the patient's age group and the parasite species. 
In areas without access to laboratory-based diagnosis:

- Case of probable uncomplicated malaria - a patient with signs and/ or symptoms of uncomplicated malaria, who receives antimalarial treatment.

- Case of probable severe malaria - a patient requiring hospitalization for signs and/or symptoms of severe malaria, who receives antimalarial treatment.

- Probable malaria death - death of a patient who has been diagnosed with probable severe malaria.

In areas with access to laboratory-based diagnosis:

- Asymptomatic malaria - laboratory confirmation (by microscopy or immunodiagnostic test) of parasitaemia in a person with no recent history of signs and/or symptoms of malaria.

- Case of confirmed uncomplicated malaria - a patient with signs and/or symptoms of uncomplicated malaria, who receives antimalarial treatment, with laboratory confirmation of diagnosis.

- Case of confirmed severe malaria - a person requiring hospitalization for signs and/or symptoms of severe malaria, who receives antimalarial treatment, with laboratory confirmation of diagnosis.

- Confirmed malaria death - death of a patient who has been diagnosed with severe malaria, with laboratory confirmation of diagnosis.

The signs and symptoms of malaria to be included in these definitions may vary in different epidemiological settings (3). The uncomplicated and severe malaria categories are intended to be mutually exclusive. For example, a patient who initially presents with uncomplicated malaria but then develops signs or symptoms of severe disease, should only be classified as having severe malaria, and not counted twice. This also applies to the probable and confirmed malaria categories. Thus, countries would be expected to report probable and confirmed cases separately.

Because of the increasing importance of antimalarial drug resistance to malaria control efforts, standardized case definition is needed for treatment failures:

- Malaria treatment failure - a patient with confirmed uncomplicated malaria with a history of having taken the correct dosage and followed the regimen of the nationally recommended antimalarial treatment, but who presents with asexual parasitaemia on a blood smear within 14 days of the start of treatment. 


\subsubsection{Indicators}

Once standardized case definitions have been agreed upon, indicators can be developed to measure the progress of the control programme (38). For the purpose of monitoring and evaluation, the indicators should be closely linked to the programme's objectives. In deciding how many indicators should be used, accurate measurement of a small number of core indicators is preferable to imprecise measurement of too many. As additional resources become available, and the programme gains experience and makes progress, these indicators can be refined, improved and added to. Most of the information needed to measure the indicators can be obtained from three general sources, although these sources may vary considerably in the quality of data they provide. The information sources are:

1. Routine data collected by the national health-information system (assuming that standardized case definitions have been agreed upon and used, and these data are of acceptable quality).

2. Interviews and/or observations in health facilities. These could be carried out during routine supervisory visits or during special surveys.

3. Specific household or community surveys. ${ }^{1}$

The first two information sources should be available to all programmes; the third will require additional programme resources. Although the cost of conducting such surveys may be high, savings can be made by measuring several indicators in the same survey.

\section{Core indicators}

Although the choice of indicators must be left up to individual national programmes, the following core (impact and outcome) indicators should be used in all malaria control programmes, irrespective of their goals or the local epidemiological situation.

\section{Impact indicators:}

- Morbidity attributed to malaria:

- number of cases of uncomplicated malaria (probable and confirmed) among target groups per unit population per unit time;

- number of cases of severe malaria (probable and confirmed) among target groups per unit population per unit time.

${ }^{1}$ Surveys may include specific questionnaires for assessing malaria control activities carried out by the community. 
- Mortality attributed to malaria:

- number of malaria deaths (probable and confirmed) among target groups per unit population per unit time;

- proportion of probable and confirmed malaria deaths among patients with severe malaria admitted to a health facility per unit time.

- Malaria treatment failures - number of microscopically confirmed malaria treatment failures per number of patients treated. These data should be reported for each drug used.

Outcome indicators:

- Availability of antimalarial drugs - percentage of health facilities reporting no disruption of the stock of antimalarial drugs (as specified in the national drug policy) during the previous 3 months.

- Reporting of morbidity and mortality indicators - percentage of districts reporting morbidity and mortality indicators to the national programme on a monthly basis during the previous 12 months.

\section{Additional indicators:}

The following additional indicators may be used, depending on the epidemiological situation and the goals of the programme.

- Annual parasite incidence (API) - number of microscopically confirmed malaria cases detected during 1 year per unit population.

- Use of insecticide-treated mosquito nets - proportion of target groups provided with insecticide-treated nets and the proportion that report that they slept under such a net the previous night. These indicators will require household or community surveys and are relevant to situations where the programme objectives are to limit and prevent transmission of falciparum malaria.

- Performance of mothers or carers - proportion of mothers or carers who ensure correct home management of children with fever, in accordance with national policies. This indicator will require household or community surveys.

- Protection of pregnant women - proportion of women in their first and second pregnancies who report per unit time that they have taken chemoprophylaxis or intermittent drug treatment, according to national drug policies.

- Preparation for malaria epidemics - proportion of epidemic-prone areas that have an epidemic containment plan and adequate stocks 
of antimalarial drugs, supplies and functioning equipment in place or easily accessible at least 1 month before the epidemic season begins. This indicator is relevant to situations where the programme objectives are to reduce mortality and morbidity, and to limit transmission and prevent epidemics of falciparum malaria.

- Intradomiciliary spraying of insecticides - proportion of houses sprayed per total number targeted for spraying. This indicator is suitable for situations where the programme objectives are to limit transmission and prevent epidemics of both falciparum and vivax malaria.

- Laboratory diagnosis:

- proportion of health districts where quality control procedures for malaria control are in place;

- proportion of health facilities which have laboratory diagnostic capabilities, from which an adequate sample of positive and negative slides have been confirmed by a reference laboratory.

Outcome indicators specific to areas where there is residual or no transmission:

- Presence of foci of transmission:

- number of villages in which autochthonous cases (classified by species) have been reported since the beginning of the previous transmission season;

- number of cases investigated (classified by species) and found to be autochthonous;

- number of malaria cases investigated.

In these areas, mixed infections should be counted as falciparum cases.

\subsection{Operational research}

The need

All national malaria control programmes must be capable of carrying out operational research so that programme activities can be made more effective and adaptable to changing epidemiological situations (1). Such research should be relevant to national programme objectives, addressing not only the efficacy of specific interventions but also social, economic, cultural and behavioural factors that may affect programme activities. Such factors can either help or hinder the efforts of health services and other collaborating sectors to implement cost-effective and sustainable malaria control, and can influence communities as they assume greater responsibility for their own protection and treatment. 
Operational research also has a part to play in the organization and delivery of malaria control. Many malarious countries are in the process of implementing economic and health sector reforms in which individuals and communities are requested to increase their contribution to health services, while the provision of free health services is reduced. Health services are also being decentralized to increase community involvement. There is a need to consider how decentralization can be used to optimize malaria control activities and manage community-based interventions.

\subsubsection{National programme capabilities for operational research}

The research capabilities of national malaria programmes vary greatly. Some countries have maintained a capability for operational research within the programme or in collaboration with national research institutes, universities and groups from industrialized countries. However, in many others, the commitment and capability to conduct research diminished as the malaria burden was reduced during the eradication era. Most malaria control programmes in Africa, south of the Sahara, have a limited capability for planning malaria control and, as a consequence, do not have a tradition of carrying out operational research. They also have limited human and financial resources. Even when these countries have national biomedical research organizations, there is often little communication or collaboration between these and the control programmes. Thus, operational research may often be divorced from the priority needs of the country's malaria situation.

\subsubsection{Constraints on operational research}

Perhaps because of the different ways in which control activities and operational research are commissioned and funded, they have taken place in relative isolation from one another in many countries. While this hinders research being directed to address programme needs and the transfer of the results into practice, other constraints on operational research also exist, such as:

- Inadequate standardization of protocols (methods, inclusion criteria and data handling) to ensure comparability and adequate analysis of results.

- Unavailability of relevant data to decision-makers. Data often remain unpublished in national and international programme reports. In addition, many national programmes have limited access to international and national journals. 
- Insufficient time for policy-makers, who are faced with urgent problems of programme implementation, to evaluate research results.

- Insufficient national expertise to identify needs at the district level, and to plan and implement operational research. Operational research tends to be focused on urgent technical issues, such as efficacy of drugs, insecticides and insecticide-impregnated materials. Although there has recently been an increased focus on social, economic and cultural issues, and health sector reform, local capacity in these research areas is particularly limited.

- Insufficient financial support at national and international levels to support operational research. Research activities are rarely incorporated into and financed as part of national plans for malaria control, relying instead on limited international and bilateral support.

- Excessive delays in changing policies in the light of new research and in the implementation of modified policies.

\subsubsection{Addressing the constraints}

Recently, there have been several initiatives to improve the coordination, implementation and funding of operational research, such as:

- WHO's initiative (1996) for accelerated implementation of malaria control in selected African countries;

- a Multinational Initiative on Malaria (1997);

- an East African Network (1997) funded from bilateral sources;

- an Asian Collaborative Training Network for Malaria (1996) in south-east Asia funded by WHO and other partners.

However, there is still an urgent need for WHO, and other partners, to advocate that operational research should be incorporated into and funded as part of all national plans of action for malaria control. This would not only help to ensure that operational research is in line with programme needs, but would also provide a system by which programmes could commission urgent operational research for which ministries of health do not, at present, have the funds.

The translation of results into practice would be facilitated at the national level if national bodies were established and/or strengthened to develop research priorities and strategies in line with control priorities. They should provide a forum for bringing together, as partners, researchers, health-care providers and policy-makers.

Tools for improving translation of results into practice are being developed. Standard protocols are now increasingly being used in operational research (39-41). Information exchange is being facili- 
tated by the development of several Web sites on the Internet, and the production of an electronic register of controlled trials by the Cochrane Infectious Disease Group (16, 42-44; see Annex 2). While access to computer-based information in malaria-endemic countries is increasing, there is still a need to increase information exchange by traditional means. In this context, WHO should publish relevant research findings as rapidly as possible and should consider strengthening and expanding the Malaria Network Web site and Internet discussion group recently established with World Bank support.

Translation of results into practice calls not only for increased dissemination of comparable research results, but also for those results to be targeted to policy-makers and those responsible for implementing control.

Training must be also an integral part of operational research interventions if national capabilities in operational research are to be increased. Priority areas include:

- development of research capabilities through hands-on involvement in protocol development and implementation, and collection, analysis and dissemination of results;

- exchange of control programme staff between endemic countries facing similar operational problems (e.g. staff from epidemic-prone areas of one country assisting another during the outbreak of an epidemic);

- modification of university curricula for health workers to address the practical needs of malaria control (e.g. aiming to increase their awareness of information, education and communication needs of the community and the patient, and the concepts of the Integrated Management of Childhood Illnesses).

\subsubsection{Priority areas for operational research related to programme objectives and policy}

Strengthening early diagnosis and prompt treatment to reduce morbidity and mortality in high-risk groups

Improving the quality of care in the home. Research issues include:

- carers' knowledge of fever (recognition of symptoms and awareness of treatment options);

- malaria prevention and the behaviour of those seeking care, and their relationship to cost and patient compliance;

- development of new approaches to improve care and management of malaria in the home, including increasing mothers' and carers' ability to recognize the symptoms of severe malaria. 
Improving disease management in the public and private sector. Research issues include:

- development of rational antimalarial drug policies, including monitoring of parasite drug resistance and therapeutic efficacy of drugs;

- improvement of the quality and provision of antimalarial drugs and health care at the peripheral level;

- relationship of the behaviour of those seeking care and other risk factors to the development of severe malaria;

- development of strategies to manage severe malaria at the periphery;

- evaluation of new diagnostic tools;

- improvement of health-worker/patient consultations (e.g. patient counselling).

\section{Strengthening capacity for implementing selective preventive measures}

Reducing the impact of malaria on pregnancy. Research issues include:

- understanding local knowledge, health-care practices and other factors (including HIV co-infection and the use of anti-HIV drugs) affecting malaria in pregnancy;

- development and evaluation of effective strategies for the protection of pregnant women.

Increasing the use of effective personal protection measures. Research issues include:

- identification of factors affecting the promotion, distribution and implementation of programmes for promoting use of impregnated bednets, particularly in Africa, south of the Sahara;

- understanding local perceptions of the use of nets for disease control and avoidance of nuisance biting;

- evaluation of the long-term impact of the use of impregnated materials on the development of vector insecticide resistance and the immune status of populations in different epidemiological situations.

Targeting selective vector control. Research issues include:

- identification of local epidemiological situations where selective vector control can be effective, and determination of the relevant contributions of different vector control methods in those settings;

- monitoring of vector insecticide resistance to assist in the development of national policies for insecticide use in disease control; 
- large-scale operational demonstrations of selective vector control;

- evaluation of different options to reduce reliance on insecticides, especially for indoor spraying.

Strengthening community involvement in malaria control

Research issues include:

- understanding local perspectives and capacities for communitybased activities;

- evaluation of different community-based approaches to determine their impact (in terms of outcome and sustainability) on high-risk groups, and their cost-effectiveness.

Strengthening national capacity to detect epidemics

Research issues include:

- development of methods to predict, detect early and prevent epidemics;

- analysis of past and current epidemics, including meteorological information, to identify causes of epidemics and develop appropriate interventions to reverse or remove underlying factors and define future actions.

Strengthening national capacity to plan, implement and evaluate malaria control

Research issues include:

- assessment of the impact of national health sector and economic reforms on the implementation of malaria control;

- management of the decentralization of control activities;

- improvement of management and evaluation procedures at district and national levels;

- evaluation of the relevance of selected impact and outcome indicators;

- development and assessment of the impact of information, education and communication materials.

\section{Award of the Darling Medal and Prize}

The Expert Committee examined the various nominations submitted for the award of the Darling Medal and Prize in conformity with Article 5 of the regulations concerned.

Having discussed in private session the relative merits of the candidates nominated, the Expert Committee, in conformity with Article 7, sent its recommendations by letter to the Director-General of WHO, who is Secretary of the Darling Foundation Committee. 


\section{Roll Back Malaria}

The governments of malaria-endemic countries have identified malaria as a high priority disease and there is growing political commitment to control it. The Director-General of WHO, accepting the challenge to lead the world's efforts against malaria, has established Roll Back Malaria (RBM), a WHO project to coordinate global actions. RBM consists of a worldwide partnership, in which all partners contribute their skills and resources to maximize the impact of $\mathrm{RBM}$ on malaria control.

The main objective of RBM is to reduce the global malaria burden significantly through interventions adapted to local needs and strengthening of the health sector.

The basic concepts of the initiative are as follows:

- RBM will address malaria as a priority health issue within the context of health sector development that promotes intersectoral collaboration and engages the community as partners.

- WHO will provide strategic support to a global partnership including organizations of the United Nations system (the United Nations Children's Fund and the United Nations Development Programme), the World Bank, national governments of malariaendemic countries, bilateral donor agencies, nongovernmental organizations and civil society.

- WHO will work as a united institution by cutting across WHO programmes within headquarters, regional and country offices to tackle malaria.

RBM will achieve its objective by:

- bringing reliable, sustainable prevention and early treatment to affected populations;

- investing in research and the development of effective and affordable tools;

- building up human and institutional resources;

- evaluating achievements against clearly defined targets.

The global partnership within RBM will:

- support governments and country partnerships;

- strengthen the health sector;

- monitor the geographical spread of the disease and measure the results of interventions;

- improve technical efficiency and capacity by building and supporting technical and research networks in malaria-endemic countries; 
- coordinate efforts of country partnerships by promoting concerted action and sharing information on malaria programmes, in order to improve resource allocation and utilization.

$\mathrm{RBM}$ is a time-limited project (5 years). It will initially focus on countries in Africa, south of the Sahara, where the malaria burden is greatest and will be extended to include other regions of the world. The preparatory phase has started: the first countries should be ready to implement RBM plans and strategies by the year 2000 .

The Expert Committee endorsed the technical basis of RBM and welcomed the initiative as a further major development in the fight against malaria.

\section{Conclusions and recommendations}

\subsection{Conclusions}

Since the endorsement of the Global Malaria Control Strategy in 1992, the validity of its principles has been confirmed throughout the world. Efforts in recent years, coordinated by WHO, to bring malaria to the forefront of the political agenda, especially in Africa, south of the Sahara, have contributed to the establishment of a sound basis for reducing the impact of malaria.

The majority of malaria-endemic countries now have malaria control programmes at various stages of implementing realistic plans of action. The implementation of these plans has resulted in a marked reduction in malaria morbidity and mortality in some countries (e.g. Brazil, Colombia, Egypt, some States in India, Oman, Thailand, Vanuatu and Viet Nam), and has allowed others (including countries in north Africa, Cyprus and Tunisia) to maintain their malaria-free status.

The lessons are clear: malaria is being controlled using the tools currently available. However, there is no room for complacency, since the greater challenge of achieving a similar impact in Africa, south of the Sahara, still has to be faced. Furthermore, the present achievements have to be sustained in the face of technical problems, including the spread of parasite drug resistance.

While existing tools have shown some impact on malaria morbidity and mortality, the most outstanding achievement has been the demonstration of a reduction in childhood mortality by the use of insecticide-impregnated mosquito nets in a range of epidemiological situations in Africa, south of the Sahara.

Experience has shown that improvements in the disease situation are sustainable only if governments maintain their commitment to 
malaria control. There is also a need to expand health services, particularly in Africa, south of the Sahara. Research and development must be continued and strengthened to provide the new tools and approaches required as the malaria situation changes. In the absence of malaria vaccines with operational impact, new drugs are especially required since the choice of drugs is limited and parasite resistance to existing drugs is increasing. Furthermore, diagnostic tools must be improved and made affordable. Thus, national governments and the private sector should be made aware of the need to invest more in malaria control efforts and of the importance of resources, to ensure a reduction in malaria morbidity and mortality.

At present, one of the main priorities in all malaria control programmes is training. Many countries lack the technical expertise - at central level to plan and evaluate the national programme, and at the intermediate and peripheral levels to manage and implement it. Many programmes do not possess adequate information systems which are sufficiently sensitive, reliable and timely to identify emerging epidemics, and to sustain the logistic capacity required to provide a rapid effective response. Particular attention must be given to train personnel at the periphery. In Africa, south of the Sahara, steps should be taken to train community members and health-care providers in the private sector.

Available information indicates that health sector reform varies from country to country, but little is known of its impact on malaria control programmes. The main concern is that indiscriminate decentralization of all functions, from policy-making to programme implementation, should be avoided. There is a need to consider which functions should be decentralized, as well as where, how and when this should happen, along with careful planning and management of these processes.

Although some additional funds to accelerate the implementation of malaria control in the countries south of the Sahara have recently become available, the needs still exceed the amount of money available. There is also no assurance that funding, at even the current level, can be continued. Therefore, the initiative by the Director-General of WHO to establish Roll Back Malaria is a welcome development. The technical basis of this project is the Global Malaria Control Strategy. Roll Back Malaria aims to cement a global partnership of malariaaffected countries, United Nations organizations, bilateral development agencies, nongovernmental organizations and the private sector, all of which are committed to a common purpose and ways of working together. The philosophy behind it is that malaria control can 
only be successful if control is considered as part of sustainable national development.

\subsection{Recommendations}

\subsubsection{General recommendation}

Relevant WHO documents should be published as quickly as possible in the appropriate WHO languages.

\subsubsection{Relationship of malaria control programmes with changing health sector reforms}

1. As ministries of health decentralize, it is essential that some national-level functions and competence in malaria control be retained or developed at the central level to guide and support the process of decentralization. This central group would provide overall programme direction and coordination, regulatory authority, and technical support and evaluation, while retaining the ability to respond rapidly and effectively to changing epidemiological situations. To ensure that experienced malaria programme staff are available at the national level, ministries of health should seek ways to create attractive opportunities for career development for their staff.

2. As part of the decentralization process, capabilities and logistic support for the implementation of malaria control activities should be ensured at the district and/or sub-district level. These should be integrated into the local health-care system but should maintain sufficient flexibility to allow a rapid and effective response to changes in the epidemiological situation.

3. Analysis of results from countries where decentralization of the health-care system and health-care financing reforms have occurred should be undertaken to give further guidance to the countries where the data were collected, as well as to countries which have not yet begun these processes.

4. Access to early diagnosis and effective treatment are essential parts of any malaria control effort, and since there is no evidence that cost recovery has improved the quality of care, the provision of free treatment for malaria should be considered for the public good. Therefore, public financial support is required as part of governments' aims to control malaria, and to ensure that antimalarial drugs are available, affordable and of high quality. Malaria programmes should also work with private sector health-care providers, making them aware of advances in knowledge about malaria and its treatment. 


\subsubsection{Disease management}

1. Given that in many endemic areas, the available antimalarial drugs are often substandard, ineffective, or sometimes only available too far from where the disease occurs, greater efforts should be made by national governments, health services and other partners to ensure that all populations at risk have easy access to antimalarial drugs which are of good quality, effective locally, affordable, and formulated and packed to optimize patient compliance. Antimalarial drugs should be accompanied by appropriate information.

2. In order to provide antimalarial drugs that are fully effective, safe, affordable and easy to administer, and likely to remain so after many years of use, pharmacokinetically compatible combinations of artemisinin drugs and other effective schizonticides should, as a matter of urgency, be developed, tested, and used in areas where the therapeutic efficacy of chloroquine is no longer satisfactory.

3. In order to improve the quality of curative care in general and to minimize the unnecessary use of antimalarial drugs, parasite detection tests (microscopical or immunological) should be made available as part of disease management at the periphery of health services in endemic and epidemic-prone areas. The need for quality control, training, sustainability and cost-effectiveness should also be taken into account.

4. Given the increasing availability of a broadening range of antimalarial drugs and formulations from a variety of sources, it is important that regulatory processes, drug quality control and quality assurance in endemic countries be improved and expanded by strengthening national capabilities and by support from an international network of technical resource centres. WHO and other international agencies should consider establishing such a network.

5. Attention should be given to training in the management of severe febrile disease in health services, including emergency measures at the primary level as well as hospital care. It is essential that such training is integrated into the broader clinical programmes, such as malaria control programmes that are an integral part of health sector reform.

\subsubsection{Drug resistance}

1. Monitoring of the efficacy of the recommended treatment options for local falciparum malaria should be a regular activity of malaria control programmes. Results of drug efficacy tests, together with costs and other operational factors, should be used to update malaria treatment guidelines as part of the national drug policy. 
2. Coordinated by WHO, international monitoring of drug resistance of $P$. falciparum should be established with the objectives of analysing the long-term trends of the spread of parasite drug resistance and sharing the information among countries. Analysis of trends will serve to monitor, evaluate and guide drug utilization strategies and other malaria control activities aimed at reducing or preventing the selection of resistance.

\subsubsection{Operational systems and operational research}

1. WHO should support the use of standardized case definitions for malaria morbidity and mortality and a limited number of core indicators to measure programme progress in all malarious countries. The use of additional indicators should be left to the discretion of individual ministries of health, based on their programme objectives and the local epidemiological situation.

2. WHO, in collaboration with its partners, should advocate the incorporation and funding of operational research, including social and economic research, as part of national malaria control programme plans of action, and the translation of the research results into practice.

3. Efforts to improve communication and interchange of information among national malaria control programmes, and between control programmes and biomedical research organizations, deserve high priority. Support for the Malaria Network Web site should be strongly encouraged.

4. Training should be an integral part of the operational research activities of national malaria control programmes, promoted through hands-on involvement in project development and implementation, data collection and analysis, and dissemination of results.

5. WHO should actively promote partnerships between health research institutions and national and international centres of excellence for general research to support multi-disciplinary operational research. The research results should be used to influence development policies outside the health sector that have an impact on the malaria situation.

\subsubsection{Malaria in pregnancy}

1. In view of the high maternal and infant morbidity and mortality associated with malaria in pregnancy, intermittent treatment with an effective, preferably one-dose antimalarial drug, provided as 
part of antenatal care, should be made available in highly endemic areas to women in their first and second pregnancies. Such intermittent treatment should be started from the second trimester onwards and should be given at intervals not less than 1 month apart.

\subsubsection{Vector control}

1. Insecticide-treated materials have been successfully and safely used to control malaria morbidity and mortality in a range of environments throughout the African and Western Pacific Regions. Therefore, it is recommended that:

(a) Large-scale operational use of insecticide-treated materials should be actively promoted, especially in areas of stable malaria transmission in Africa, south of the Sahara.

(b) Various options for the promotion, distribution, treatment and re-treatment of materials should be investigated to address issues for adequate coverage, equity and sustainability.

(c) Evaluation and surveillance of bednet use should be an integral component of malaria control.

2. Although impregnated materials have been proven to have an impact on overall mortality in the short term, their long-term effects on malaria morbidity and mortality still need to be investigated.

3. Pyrethroids are increasingly used in vector control and are currently the only compounds available for bednet treatment. Crossresistance to a wide range of pyrethroids has already been reported in some of the major malaria vectors. Therefore, it is recommended that:

(a) Insecticide resistance in malaria vectors should be carefully monitored. This should preferably be carried out in the networks designed to build up technical expertise at country level to provide guidance for appropriate use of available insecticides and management of vector resistance.

(b) The search for non-pyrethroid alternatives should be stimulated.

4. Indoor residual spraying will continue to be of particular importance in some epidemiological settings, including the prevention and control of epidemics. Countries where vector control currently relies primarily on indoor residual spraying should focus on further selective use of this tool by improving surveillance mechanisms and carrying out rapid, detailed analysis of the data collected. 
5. WHO should provide assistance to Member States in developing appropriate mechanisms for improving the targeting of vector control interventions according to different epidemiological situations, taking into account various existing managerial structures involved in malaria control. Integrated and selective vector control will reduce reliance on chemical insecticides and address sustainability issues.

\subsubsection{DDT}

1. WHO, in its capacity of observer, should strengthen its active engagement in the work of the Intergovernmental Negotiating Committee, and call attention to the implications for malaria vector control of any type of regulation of DDT use that may be proposed by a future Persistent Organic Pollutants Convention.

2. WHO should assist the appropriate vector control structures for public health programmes of ministries of health in Member States, through a process of consultation and information dissemination, to participate actively in the national activities related to the work of the Intergovernmental Negotiating Committee leading up to a future Persistent Organic Pollutants Convention.

3. It is anticipated that for some time to come there will continue to be a role for DDT in combating malaria, particularly in the poorest endemic countries. Restrictions on DDT for public health use contained in a future Persistent Organic Pollutants Convention should therefore be accompanied by technical and financial mechanisms to ensure that effective malaria control is maintained, to at least the same level, through vector control methods that depend less on pesticides generally, and on DDT in particular.

\subsubsection{Epidemics}

1. The effectiveness of epidemic prevention and control is directly proportional to the level of preparedness of the health services and the functioning of the prediction system. Governments should give full support to the strengthening or development of such services, with the collaboration, if needed, of WHO and external agencies. In particular, these services should identify and demarcate their epidemic-prone areas, recognize the local determinants of epidemics as well as risk indicators, and start the collection and analysis of relevant data.

2. Collaboration mechanisms with other important sectors (e.g. meteorological and agricultural services) should be established to facilitate detection of epidemic risk. Malaria epidemiologists 
should also support district medical officers in maintaining appropriate records and in guiding communities in the early detection and control of malaria epidemics.

3. WHO should coordinate existing initiatives for early detection and control of epidemics, as well as the appropriate use of information and resources across interregional and international borders.

4. Countries with no or sporadic transmission should identify their malaria-susceptible areas and monitor importation of malaria cases in order to prevent re-establishment of transmission.

\section{Acknowledgements}

The Committee wishes to acknowledge the special contributions made during its deliberations by the following: Dr K. Behbehani, Division of Control of Tropical Diseases, WHO, Geneva, Switzerland; Dr A.E. Beljaev, WHO Regional Office for the Eastern Mediterranean, Alexandria, Egypt; Dr F. Binka, Roll Back Malaria, WHO, Geneva, Switzerland; Mr R. Bos, Division of Operational Support in Environmental Health, WHO, Geneva, Switzerland; Dr A. Bosman, Malaria Control, Division of Control of Tropical Diseases, WHO, Geneva, Switzerland; Dr M. Cham, Malaria Control, Division of Control of Tropical Diseases, WHO, Geneva, Switzerland; Mr J. Cheyne, Programme for Resource Mobilization, WHO, Geneva, Switzerland; Dr M.R. Couper, Division of Drug Management and Policies, WHO, Geneva, Switzerland; Mr A.L. Creese, Division of Analysis, Research and Assessment, WHO, Geneva, Switzerland; Dr C. Delacollette, Malaria Control, Division of Control of Tropical Diseases, WHO, Geneva, Switzerland; Dr H. Engers, Product Research and Development, UNDP/World Bank/WHO Special Programme for Research and Training in Tropical Diseases, WHO, Geneva, Switzerland; Dr D. Evans, Global Programme on Evidence for Policy, WHO, Geneva, Switzerland; Dr T. Godal, Roll Back Malaria, WHO, Geneva, Switzerland; Dr R.J. Guidotti, Division of Reproductive Health, Maternal and Newborn Health/Safe Motherhood, WHO, Geneva, Switzerland; Dr R. Gusmão, WHO Regional Office for the Americas/Pan American Sanitary Bureau, Washington, D.C., USA; Mr G. Hartl, Communicable Diseases, WHO, Geneva, Switzerland; Dr P. Herath, Malaria Control, Division of Control of Tropical Diseases, WHO, Geneva, Switzerland; Dr D.L. Heymann, Communicable Diseases, WHO, Geneva, Switzerland; Dr H.M. Kahssay, Division of Intensified Cooperation with Countries and Peoples in Greatest Need, WHO, Geneva, Switzerland; Dr Y. Kassankogno, WHO Regional Office for Africa, Harare, Zimbabwe; Dr J. Liljestrand, Division of Reproductive Health, Maternal and Newborn Health/Safe Motherhood, WHO, Geneva, Switzerland; Dr S. Lwanga, Strengthening Country Health Information, WHO, Geneva, Switzerland; Dr L. Manga, WHO Regional Office for Africa, Harare, Zimbabwe; Dr. K.N. Mendis, Roll Back Malaria, WHO, Geneva, Switzerland; Dr C. Morel, UNDP/World Bank/WHO Special Programme for Research and Training in Tropical Diseases, WHO, Geneva, Switzerland; Dr M. Nathan, Filariasis Control, Division of Control of Tropical Diseases, WHO, Geneva, Switzerland; Dr P.L. Olliaro, Product Research and Development, UNDP/World Bank/WHO Special Programme for Research and Training in Tropical Diseases, WHO, Geneva, Switzerland; Dr V.S. Orlov, WHO Regional Office for South-East Asia, New Delhi, India; Dr A. Rietveld, Malaria Control, Division of Control of Tropical Diseases, WHO, Geneva, Switzerland; Dr A. Robb, WHO Regional Office for Africa, Harare, Zimbabwe; Dr D. Robinson, Division of Child Health and Development, WHO, Geneva, Switzerland; Dr G. 
Sabatinelli, WHO Regional Office for Europe, Copenhagen, Denmark; $\operatorname{Dr}$ A. Schapira, Office of the WHO Representative in the Socialist Republic of Viet Nam, Hanoi, Viet Nam; Dr A. Teklehaimanot, Malaria Control, Division of Control of Tropical Diseases, WHO, Geneva, Switzerland; Dr P. Trigg, Malaria Control, Division of Control of Tropical Diseases, WHO, Geneva, Switzerland; Dr O. Walker, WHO Regional Office for Africa, Harare, Zimbabwe; Mr K. Woldeyesus, Action Programme on Essential Drugs, WHO, Geneva, Switzerland; and Dr M. Zaim, WHO Pesticide Evaluation Scheme, Division of Control of Tropical Diseases, WHO, Geneva, Switzerland.

The Committee also expresses its thanks to Professor M.E. Molyneux and Dr D. Payne for their contribution to the discussions through their working papers.

In addition, the Committee would like to express its gratitude to Ms J. Curry for organizing the meeting and to Mrs J. Griffith and Mrs P. Griffiths for providing support services.

\section{References}

1. A global strategy for malaria control. Geneva, World Health Organization, 1993.

2. Integrated management of childhood illness. Geneva, World Health Organization, 1997 (unpublished document WHO/CHD/97.3. A-L; available on request from Child and Adolescent Health and Development, World Health Organization, 1211 Geneva 27, Switzerland).

3. Implementation of the Global Malaria Control Strategy. Report of a WHO Study Group on the Implementation of the Global Plan of Action for Malaria Control 1993-2000. Geneva, World Health Organization, 1993 (WHO Technical Report Series, No. 839).

4. Management of uncomplicated malaria and the use of antimalarial drugs for the protection of travellers: report of an informal consultation, Geneva, 1821 September 1995. Geneva, World Health Organization, 1997 (unpublished document WHO/MAL/96.1075 Rev. 1; available on request from the Documentation Centre, Communicable Diseases, World Health Organization, 1211 Geneva 27, Switzerland).

5. Marsh K et al. Clinical algorithm for malaria in Africa. Lancet, 1996, 347:1327-1329.

6. Redd SC et al. Clinical algorithm for treatment of Plasmodium falciparum malaria in children. Lancet, 1996, 347:223-227.

7. Olaleye BO et al. Clinical predictors of malaria in Gambian children with fever or a history of fever. Transactions of the Royal Society of Tropical Medicine and Hygiene, 1998, 92:300-304.

8. Genton B et al. Malaria: how useful are clinical criteria for improving the diagnosis in a highly endemic area? Transactions of the Royal Society of Tropical Medicine and Hygiene, 1994, 88:537-541.

9. Weber MW et al. Pallor as a clinical sign of severe anaemia in children: an investigation in the Gambia. Bulletin of the World Health Organization, 1997, 75(Suppl. 1):113-118. 
10. Beadle $\mathrm{C}$ et al. Diagnosis of malaria by detection of Plasmodium falciparum HRP-2 antigen with a rapid dipstick antigen-capture assay. Lancet, 1994, 343:564-568.

11. Shiff CJ, Premji Z, Minjas JN. The rapid manual ParaSight-F test. A new diagnostic tool for Plasmodium falciparum infection. Transactions of the Royal Society of Tropical Medicine and Hygiene, 1993, 87:646-648.

12. Makler MT, Palmer CJ, Ager AL. A review of practical techniques for the diagnosis of malaria. Annals of tropical medicine and parasitology, 1998, 92:419-433.

13. Gilles HM, Warrell DA, eds. Bruce-Chwatt's essential malariology, 3rd ed. London, Arnold, 1993.

14. Practical chemotherapy of malaria. Report of a WHO Scientific Group. Geneva, World Health Organization, 1990 (WHO Technical Report Series, No. 805).

15. Antimalarial drug policies: data requirements, treatment of uncomplicated malaria and management of malaria in pregnancy. Report of an informal consultation, Geneva, 14-18 March 1994. Geneva, World Health Organization, 1994 (unpublished document WHO/MAL/94.1070; available on request from the Documentation Centre, Communicable Diseases, World Health Organization, 1211 Geneva 27, Switzerland).

16. Olliaro PL et al. Systematic review of amodiaquine treatment in uncomplicated malaria. Lancet, 1996, 348:1196-1201.

17. Warsame $M$ et al. Susceptibility of Plasmodium falciparum in vitro to chloroquine, mefloquine, quinine and sulfadoxine/pyrimethamine in Somalia: relationships between the responses to the different drugs. Transactions of the Royal Society of Tropical Medicine and Hygiene, 1991, 85:565-569.

18. The use of artemisinin and its derivatives as anti-malarial drugs. Report of a Joint CTD/DMP/TDR Informal Consultation, Geneva, 10-12 June 1998.

Geneva, World Health Organization, 1998 (unpublished document WHO/ MAL/98.1086; available on request from the Documentation Centre, Communicable Diseases, World Health Organization, 1211 Geneva 27, Switzerland).

19. Mwenesi H, Harpham T, Snow RW. Child malaria treatment practices among mothers in Kenya. Social science and medicine, 1995, 40:12711277.

20. Management of severe malaria: a practical handbook, 2nd ed. Geneva, World Health Organization, 2000.

21. Assessment of therapeutic efficacy of antimalarial drugs: for uncomplicated falciparum malaria in areas with intense transmission. Geneva, World Health Organization, 1996 (unpublished document WHO/MAL/96.1077; available on request from the Documentation Centre, Communicable Diseases, World Health Organization, 1211 Geneva 27, Switzerland).

22. Mackinnon MJ, Hastings IM. The evolution of multiple drug resistance in malaria parasites. Transactions of the Royal Society of Tropical Medicine and Hygiene, 1998, 92:188-195. 
23. Ray AP et al. P. falciparum Containment Programme. Ten years of operation in India (1978-1988). Delhi, P. falciparum Containment Programme/

Directorate of National Malaria Control Programme/World Health

Organization/Swedish International Development Authority, 1988:236-239.

24. White NJ. Preventing antimalarial drug resistance through combinations. Drug resistance updates, 1988, 1:3-9.

25. Baird JK et al. Primaquine for prophylaxis against malaria among nonimmune transmigrants in Irian Jaya, Indonesia. American journal of tropical medicine and hygiene, 1995, 52:479-484.

26. Vector control for malaria and other mosquito-borne diseases. Report of a WHO Study Group. Geneva, World Health Organization, 1995 (WHO Technical Report Series, No. 857).

27. Agenda 21, the United Nations programme of action from Rio. New York, United Nations, 1993.

28. Price RN et al. Effects of artemisinin derivatives on malaria transmissibility. Lancet, 1996, 347:1654-1658.

29. International travel and health: vaccination requirements and health advice: situation as on 1 January 1998. Geneva, World Health Organization, 1998.

30. Parise ME et al. Efficacy of sulfadoxine-pyrimethamine for prevention of placental malaria in an area of Kenya with a high prevalence of malaria and human immunodeficiency virus infection. American journal of tropical medicine and hygiene, 1998, 59:813-822.

31. Verhoeff FH et al. An evaluation of the effects of intermittent sulfadoxinepyrimethamine treatment in pregnancy on parasite clearance and risk of low birthweight in rural Malawi. Annals of tropical medicine and parasitology, 1998, 92:141-150.

32. Schultz LJ et al. The efficacy of antimalarial regimens containing sulfadoxine-pyrimethamine and/or chloroquine in preventing peripheral and placental Plasmodium falciparum infection among pregnant women in Malawi. American journal of tropical medicine and hygiene, 1994, 51:515522.

33. Shulman CE et al. Intermittent sulfadoxine-pyrimethamine to prevent severe anaemia secondary to malaria in pregnancy: a randomised placebocontrolled trial. Lancet, 1999, 353:632-636.

34. Brabin BJ et al. Antimalarial drug policy in Malawi. Annals of tropical medicine and parasitology, 1997(Suppl. 1):S113-S115.

35. Brabin B. An assessment of low birthweight risk in primiparae as an indicator of malaria control in pregnancy. International journal of epidemiology, 1991, 20:276-283.

36. Verhoeff $\mathrm{FH}$ et al. Increased prevalence of malaria in HIV-infected pregnant women and its implications for malaria control. Journal of tropical medicine and international health, 1999, 4:5-12.

37. Steketee RW et al. Impairment of a pregnant women's acquired ability to limit Plasmodium falciparum by infection with human immunodeficiency virus type-1. American journal of tropical medicine and hygiene, 1996,

55(1 Suppl):42-49. 
38. Information systems for the evaluation of malaria control programmes: a practical guide. Brazzaville, WHO Regional Office for Africa, 1994

(unpublished document AFRO/CTD/MAL/94.3; available on request from the Documentation Centre, Communicable Diseases, World Health Organization, 1211 Geneva 27, Switzerland).

39. Carnevale $\mathbf{P}$ et al. Un protocole simplifié pour l'évaluation des pratiques et des coûts de la lutte antivectorielle faite à l'échelon individuel et familial. [A simplified protocol for evaluation of vector control costs and practices at individual and family levels.] Geneva, World Health Organization, 1997 (unpublished document WHO/MAL/97.1078; available on request from the Documentation Centre, Communicable Diseases, World Health Organization, 1211 Geneva 27, Switzerland).

40. Kikwawila Study Group Qualitative research methods: teaching materials from a TDR Workshop. Social and Economic Research in Tropical diseases Resource Paper No. 3. Geneva, World Health Organization, 1994 (unpublished document TDR/SER/RP/94.2; available on request from Tropical Diseases Research Communications, World Health Organization, 1211 Geneva 27, Switzerland).

41. Status of the accelerated implementation of malaria control activities in Africa. Summary of mid-term review reports. Geneva, World Health Organization, 1998 (unpublished document CTD/MAL/AFRO/EMRO/98.5; available on request from the Documentation Centre, Communicable Diseases, World Health Organization, 1211 Geneva 27, Switzerland).

42. Lengeler C, Cattani J, de Savigny D, eds. Net gain: a new method for preventing malaria deaths. Ottawa, International Development Research Centre/World Health Organization, 1996.

43. Mclntosh HM, Greenwood BM. Chloroquine or amodiaquine combined with sulfadoxine-pyrimethamine as a treatment for uncomplicated malaria - a systematic review. Annals of tropical medicine and parasitology, 1998, 92:265-270.

44. Garner P et al. eds. Infectious diseases module of the Cochrane database of systematic reviews, 1997 (available from the Cochrane library [Web site: http://www.updateusa.com/clibip/clib.htm]). 


\section{Annex 1 \\ Use of DDT in vector control ${ }^{1}$}

The WHO Study Group on Vector Control for Malaria and Other Mosquito-Borne Diseases considered the current situation regarding the use of DDT for controlling vector-borne diseases, in particular malaria, in the light of two recent publications suggesting an association between DDT and human cancers $(1,2)$, a report on the presence of DDT in breast milk (3), and two general reviews of the subject. ${ }^{2}$ Two expert toxicologists ${ }^{3}$ were invited to review these papers, including the citations, and to participate in the discussions on DDT.

After careful review of the documents and intensive discussion, the Study Group concluded that:

1. The information presented does not provide convincing evidence of adverse effects of DDT exposure as a result of indoor residual spraying as carried out in malaria control activities.

2. There is therefore, at this stage, no justification on toxicological or epidemiological grounds for changing current policy (4) towards indoor spraying of DDT for vector-borne disease control.

3. DDT may therefore be used for vector control, provided that all the following conditions are met:

(a) it is used only for indoor spraying;

(b) it is effective;

(c) the material is manufactured to the specifications issued by WHO (5);

(d) the necessary safety precautions are taken in its use and disposal.

4. In considering whether to use DDT, governments should take into account the following additional factors:

(a) the costs involved in the use of insecticides (DDT or alternatives);

(b) the role of insecticides in focal or selective vector control, as specified in the Global Malaria Control Strategy $(6,7)$;

(c) the availability of alternative vector control methods, including alternative insecticides (in view of the availability of

\footnotetext{
${ }^{1}$ Reproduced, with minor editorial changes, from Vector control for malaria and other mosquito-borne diseases. Report of a WHO Study Group. Geneva, World Health Organization, 1995 (WHO Technical Report Series, No. 857).

2 Prepared by: Dr C.F. Curtis, Department of Medical Parasitology, London School of Hygiene and Tropical Medicine, London, England; and Professor J. Mouchet, French Institute for Cooperative Scientific Research for Development (ORSTOM), Paris, France.

${ }^{3}$ Dr W.N. Aldridge, The Robens Institute, University of Surrey, Guildford, England; and Professor M. Lotti, Institute of Occupational Medicine, University of Padua, Padua, Italy.
} 
alternative insecticides for indoor residual spraying, some of which may compete with DDT in terms of epidemiological impact, public acceptability, logistic suitability and compliance with specifications issued by WHO, DDT no longer merits being considered the only insecticide of choice);

(d) the implications for insecticide resistance, including possible cross-resistance to some alternative insecticides;

(e) the changing public attitude to pesticide use, including public health applications.

5. Given the paucity of data suggesting adverse effects of indoor house-spraying, further epidemiological investigation using rigorous scientific protocols is to be encouraged.

6. Further studies should also be carried out to:

(a) examine the health effects of DDT in breast milk on breastfed infants, including any resulting behavioural change;

(b) investigate thoroughly any suspected association between the use of DDT in routine malaria control activities and an increased incidence of cancer(s);

(c) clarify the significance of the reduction in muscarinic receptor density caused by DDT.

\section{References}

1. Garabrant DH et al. DTT and related compounds and risk of pancreatic cancer. Journal of the National Cancer Institute, 1992, 84:764-771.

2. Wolff MS et al. Blood levels of organochlorine residues and risk of breast cancer. Journal of the National Cancer Institute, 1993, 85:648-652.

3. Bouwman $\mathrm{H}$ et al. Levels of DDT and metabolites in breast milk from Kwa-Zulu mothers after DDT application for malaria control. Bulletin of the World Health Organization, 1990, 68:761-768.

4. The place of DDT in operations against malaria and other vector-borne diseases. In: Executive Board Forty-seventh Session, Geneva, 19-29 January 1971, Part II. Report on the proposed programme and budget estimates for 1972. Geneva, World Health Organization, 1971 (Official Records of the World Health Organization, No. 190):176-182.

5. Specifications for pesticides used in public health, 7th ed. Geneva, World Health Organization, 1997 (unpublished document WHO/CTD/WHOPES/97.1; available on request from the Documentation Centre, Communicable Diseases, World Health Organization, 1211 Geneva 27, Switzerland).

6. A global strategy for malaria control. Geneva, World Health Organization, 1993.

7. Implementation of the Global Malaria Control Strategy. Report of a WHO Study Group on the Implementation of the Global Plan of Action for Malaria Control 1993-2000. Geneva, World Health Organization, 1993 (WHO Technical Report Series, No. 839). 


\section{Annex 2 \\ Improving information exchange}

There have been several initiatives in recent years to increase the rapid exchange of information on malaria. These include the:

- Malaria Network, a Web site and Internet discussion group established in June 1998 by WHO in collaboration with the World Bank. This is aimed at malaria control managers, ministry of health staff and other health workers with special responsibilities for the implementation of malaria control. It provides technical and managerial information relevant for malaria control activities in the field and functions as a forum for discussion. The network is demand-driven, and is regularly adapted to the identified needs of the target usergroups. Web site: http://www.malarianetwork.org.

- Malaria Foundation International: Global Networking Against Malaria. Founded in 1992 to facilitate the development and implementation of solutions to the health, economic and social problems caused by malaria, by supporting global communication and networking, research, education and training. The Web site includes databases on the malaria research network and malaria genome, and provides updated information on all aspects of malaria and its control. Web site: http://www.malaria.org.

- Multilateral Initiative on Malaria (MIM) Newsletter, published by the Wellcome Trust since mid-1998 to provide information on the current activities of the Initiative. It is also available on the Wellcome Trust's Web site: http://www.wellcome.ac.uk.

- Asian Collaborative Training Network for Malaria (ACTMalaria) Web site. The ACTMalaria newsletter is available at this site: http: //www.actmalaria.org.

- Environmental Health Project, which has a special bibliography of malaria-related articles, including publications on bednets. Web site: http://www.access.digex.net/ ehp/webliog-html.

- Cochrane Infectious Disease Group maintains an electronic register of controlled trials, available on CD-ROM and diskette. Cochrane library Web site: http://www.updateusa.com/clibip/clib.htm. 


\section{World Health Organization Technical Report Series}

Recent reports:

No

Sw.fr.*

823 (1992) WHO Expert Committee on Specifications for Pharmaceutical

Preparations

Thirty-second report (140 pages)

17.-

824 (1992) WHO Expert Committee on Rabies

Eighth report (90 pages)

825 (1992) The use of essential drugs

Fifth report of the WHO Expert Committee (79 pages) 10.-

826 (1992) Recent advances in oral health

Report of a WHO Expert Committee (42 pages)

827 (1992) The role of health centres in the development of urban health systems

Report of a WHO Study Group on Primary Health Care in Urban Areas

(42 pages)

828 (1992) Evaluation of certain food additives and naturally occurring toxicants

Thirty-ninth report of the Joint FAO/WHO Expert Committee on Food

Additives (57 pages)

829 (1993) Evaluation of recent changes in the financing of health services

Report of a WHO Study Group (79 pages)

830 (1993) The control of schistosomiasis

Second report of the WHO Expert Committee (93 pages)

7.-

7.-

9.-

10.-

12.-

831 (1993) Rehabilitation after cardiovascular diseases, with special emphasis on developing countries

Report of a WHO Expert Committee (130 pages)

17.-

832 (1993) Evaluation of certain veterinary drug residues in food

Fortieth report of the Joint FAO/WHO Expert Committee on Food Additives (68 pages)

833 (1993) Health promotion in the workplace: alcohol and drug abuse

Report of a WHO Expert Committee (39 pages)

834 (1993) WHO Expert Committee on Specifications for Pharmaceutical

Preparations

Thirty-third report (35 pages)

835 (1993) Aging and working capacity

Report of a WHO Study Group (55 pages)

836 (1993) WHO Expert Committee on Drug Dependence

Twenty-eighth report (50 pages)

837 (1993) Evaluation of certain food additives and contaminants

Forty-first report of the Joint FAO/WHO Expert Committee on Food

Additives (61 pages)

838 (1993) Increasing the relevance of education for health professionals

Report of a WHO Study Group on Problem-Solving Education for the Health Professions (33 pages)

839 (1993) Implementation of the Global Malaria Control Strategy

Report of a WHO Study Group on the Implementation of the Global Plan of Action for Malaria Control 1993-2000 (62 pages)

840 (1994) WHO Expert Committee on Biological Standardization

Forty-third report (223 pages)

10.-

7.-

7.-

10.-

$10 .-$

10.-

8.-

10.-

31.-

841 (1994) Cardiovascular disease risk factors: new areas for research

Report of a WHO Scientific Group (59 pages)

10.-

842 (1994) Nursing beyond the year 2000

Report of a WHO Study Group (25 pages)

6.-

* Prices in developing countries are $70 \%$ of those listed here. 
843 (1994) Assessment of fracture risk and its application to screening for

postmenopausal osteoporosis

Report of a WHO Study Group (134 pages) 22.-

844 (1994) Prevention of diabetes mellitus

Report of a WHO Study Group (108 pages)

845 (1994) Information support for new public health action at district level

Report of a WHO Expert Committee (35 pages) 8.-

846 (1994) Fluorides and oral health

Report of a WHO Expert Committee on Oral Health Status and Fluoride Use

(42 pages)

847 (1994) Chemotherapy of leprosy

Report of a WHO Study Group (29 pages)

848 (1994) WHO Expert Committee on Biological Standardization

Forty-fourth report (94 pages)

849 (1995) Control of foodborne trematode infections

Report of a WHO Study Group (165 pages) 26.-

850 (1995) The use of essential drugs

Sixth report of the WHO Expert Committee (144 pages)

851 (1995) Evaluation of certain veterinary drug residues in food

Forty-second report of the Joint FAO/WHO Expert Committee on Food

Additives (50 pages)

852 (1995) Onchocerciasis and its control

Report of a WHO Expert Committee on Onchocerciasis Control (111 pages) 15.-

853 (1995) Epidemiology and prevention of cardiovascular diseases in elderly people

Report of a WHO Study Group (72 pages)

854 (1995) Physical status: the use and interpretation of anthropometry

Report of a WHO Expert Committee (462 pages)

855 (1995) Evaluation of certain veterinary drug residues in food

Forty-third report of the Joint FAO/WHO Expert Committee on Food

Additives (65 pages)

856 (1995) WHO Expert Committee on Drug Dependence

Twenty-ninth report (21 pages)

857 (1995) Vector control for malaria and other mosquito-borne diseases

Report of a WHO Study Group (97 pages)

858 (1995) WHO Expert Committee on Biological Standardization

Forty-fifth report (108 pages)

859 (1995) Evaluation of certain food additives and contaminants

Forty-fourth report of the Joint FAO/WHO Expert Committee on Food

Additives (62 pages)

860 (1996) Nursing practice

Report of a WHO Expert Committee (37 pages) 12.-

861 (1996) Integration of health care delivery

Report of a WHO Study Group (74 pages)

862 (1996) Hypertension control

Report of a WHO Expert Committee (89 pages)

863 (1996) WHO Expert Committee on Specifications for Pharmaceutical

Preparations

Thirty-fourth report (200 pages)

864 (1996) Evaluation of certain veterinary drug residues in food

Forty-fifth report of the Joint FAO/WHO Expert Committee on Food

Additives (66 pages)

16.-

(1996) Control of hereditary diseases

Report of a WHO Scientific Group (89 pages)

12.-

6.-

15.-

17.-

11.-

35.-

(1996) Research on the memopause in the 1990s

Report of a WHO Scientific Group (114 pages)

(1997) The use of essential drugs

Seventh report of the WHO Expert Committee (80 pages) 
868 (1997) Evaluation of certain food additives and contaminants

Forty-sixth report of the Joint FAO/WHO Expert Committee on Food

Additives (77 pages)

14.-

869 (1997) Improving the performance of health centres in district health systems

Report of a WHO Study Group (70 pages)

870 (1997) Promoting health through schools

Report of a WHO Expert Committee on Comprehensive School Health

Education and Promotion (99 pages)

871 (1997) Medical methods for termination of pregnancy

Report of a WHO Scientific Group (117 pages)

872 (1998) WHO Expert Committee on Biological Standardization

Forty-sixth report (97 pages)

873 (1998) WHO Expert Committee on Drug Dependence

Thirtieth report (56 pages)

874 (1998) WHO Expert Committee on Leprosy

Seventh report (49 pages)

875 (1998) Training in diagnostic ultrasound: essentials, principles and standards Report of a WHO Study Group (52 pages)

876 (1998) Evaluation of certain veterinary drug residues in food

Forty-seventh report of the Joint FAO/WHO Expert Committee on Food

Additives (91 pages)

877 (1998) Cardiovascular disease and steroid hormone contraception

Report of a WHO Scientific Group (96 pages)

878 (1998) WHO Expert Committee on Biological Standardization

Forty-seventh report (107 pages)

879 (1998) Evaluation of certain veterinary drug residues in food

Forty-eighth report of the Joint FAO/WHO Expert Committee on Food

Additives (80 pages)

880 (1998) Preparation and use of food-based dietary guidelines

Report of a Joint FAO/WHO Consultation (114 pages)

881 (1998) Control and surveillance of African trypanosomiasis

Report of a WHO Expert Committee (119 pages)

882 (1998) The use of essential drugs

Eighth report of the WHO Expert Committee (83 pages)

883 (1999) Food safety issues associated with products from acquaculture

Report of a Joint FAO/NACA/WHO Study Group (63 pages)

884 (1999) Evaluation of certain food additives and contaminants

Forty-ninth report of the Joint FAO/WHO Expert Committee on Food

Additives (104 pages)

885 (1999) WHO Expert Committee on Specifications for Pharmaceutical

Preparations

Thirty-fifth report (162 pages)

886 (1999) Programming for adolescent health and development

Report of a WHO/UNFPA/UNICEF Study Group (266 pages)

887 (1999) WHO Expert Committee on Drug Dependence

Thirty-first report (28 pages)

888 (1999) Evaluation of certain veterinary drug residues in food

Fiftieth report of the Joint FAO/WHO Expert Committee on Food Additives

(102 pages)

889 (1999) WHO Expert Committee on Biological Standardization

Forty-eighth report (117 pages)

890 (1999) High-dose irradiation: wholesomeness of food irradiated with doses

above 10kGy

Report of a Joint FAO/IAEA/WHO Study Group (203 pages)

891 (2000) Evaluation of certain food additives

Fifty-first report of the Joint FAO/WHO Expert Committee on Food

Additives
14.-

20.-

23.-

20.-

14.-

14.-

14.-

19.-

20.-

16.-

23.-

23.-

19.-

14.-

20.-

35.-

56.-

14.-

20.-

23.-

42.- 


\section{SELECTED WHO PUBLICATIONS OF RELATED INTEREST}

Price (Sw.fr.)*

Implementation of the Global Malaria Control Strategy.

Report of a WHO Study Group on the Implementation of the

Global Plan of Action for Malaria Control 1993-2000.

WHO Technical Report Series, No. 839, 1993 (62 pages)

Bench aids for the diagnosis of malaria infections.

L.R. Ash et al. (in press)

Basic malaria microscopy.

Part I. Learner's guide. 1991 (72 pages)

Part II. Tutor's guide. 1991 (69 pages)

Entomological field techniques for malaria control.

Part I. Learner's guide. 1992 (77 pages)

Part II. Tutor's guide. 1992 (54 pages)

Practical chemotherapy of malaria.

Report of a WHO Scientific Group.

WHO Technical Report Series, No. 805, 1990 (158 pages)

Management of severe malaria: a practical handbook, $2 \mathrm{nd}$ ed.

2000 (77 pages)

Malaria: a manual for community health workers

1996 (55 pages)

Vector control for malaria and other mosquito-borne diseases.

Report of a WHO Study Group.

WHO Technical Report Series, No. 857, 1995 (97 pages)

Vector control: methods for use by individuals and communities.

Prepared by J.A. Rozendaal.

1997 (424 pages)

Further information on these and other WHO publications can be obtained from Marketing and Dissemination, World Health Organization, 1211 Geneva 27, Switzerland 
Despite considerable progress in malaria control over the past decade, malaria remains a serious problem - particularly in Africa, south of the Sahara, where about $90 \%$ of clinical cases occur. Malaria, either alone or in combination with other diseases, is estimated to kill between 1.1 and 2.7 million people worldwide each year, and over 2400 million remain at risk.

This report of a WHO Expert Committee reviews the progress made since 1992 in the implementation of the Global Malaria Control Strategy and analyses the effect of health sector reforms on malaria control programmes. The importance of more recent initiatives, such as the Roll Back Malaria project, is also discussed.

The report focuses on disease management and on drug resistance of malaria parasites, one of the greatest challenges currently facing control programmes. Guidance is given on how to predict, prepare for, control and prevent malaria epidemics, which threaten large areas of the world.

The use of chemoprophylaxis and selective vector control in the prevention of malaria is discussed. As accurate epidemiological information is essential for assessing public health needs and for monitoring malaria control programmes, a number of standardized case definitions and indicators have been developed. In order to make programme activities effective and responsive to changing epidemiological situations, the report emphasizes the need for operational research at a national level.

Although existing tools have had an impact on malaria morbidity and mortality, it is clear that much remains to be done. The report concludes with a list of recommendations to ensure that the present achievements are sustained and that a greater impact is made on the disease, particularly in Africa. 